\title{
Numerical solution of fuzzy boundary value problems using Galerkin method
}

\author{
SMITA TAPASWINI ${ }^{1,2, *}$, S CHAKRAVERTY $^{3}$ and JUAN J NIETO ${ }^{4,5}$ \\ ${ }^{1}$ College of Mathematics and Statistics, Chongqing University, Chongqing 401331, China \\ ${ }^{2}$ Department of Mathematics, Kalinga Institute of Industrial Technology, Bhubaneswar, Odisha 751 024, India \\ ${ }^{3}$ Department of Mathematics, National Institute of Technology, Rourkela, Odisha 769 008, India \\ ${ }^{4}$ Department of Mathematics, Faculty of Science, King Abdulaziz University, P.O. Box 80203, Jeddah 21589, \\ Saudi Arabia \\ ${ }_{5}^{5}$ Department of Mathematical Analysis, Faculty of Mathematics, University of Santiago de Compostela, \\ 15782 Santiago de Compostela, Spain \\ e-mail: smitatapaswini@gmail.com; sne_chak@yahoo.com; juanjose.nieto.roig@usc.es
}

MS received 4 September 2015; revised 27 January 2016; accepted 6 September 2016

\begin{abstract}
This paper proposes a new technique based on Galerkin method for solving $n$th order fuzzy boundary value problem. The proposed method has been illustrated by considering three different cases depending upon the sign of coefficients with benchmark example problems. To show the applicability of the proposed method, an application problem related to heat conduction has also been studied. The results obtained by the proposed methods are compared with the exact solution and other existing methods for demonstrating the validity and efficiency of the present method.
\end{abstract}

Keywords. Fuzzy number; triangular fuzzy number; $n$th order fuzzy boundary value problems; Galerkin method.

\section{Introduction}

In recent years, the study of fuzzy differential equations (FDEs) has been expanding rapidly as a new branch of fuzzy mathematics. The concept of fuzzy set theory was first developed by Zadeh [1]. FDEs play an important role for modelling physical and engineering problems because they may mimic the real situation to handle the systems under uncertainty. But, it is too difficult to obtain exact solutions of FDEs, due to the complexity in fuzzy arithmetic. For example, addition is not the inverse operation of subtraction. In a similar manner multiplication is not the inverse operation of division too. Hence, one may need reliable and efficient numerical techniques to handle the corresponding FDEs.

There exist a variety of papers dealing with FDEs and their applications. Some of these are reviewed and cited here for better understanding of the present investigation. Chang and Zadeh [2] first introduced the concept of a fuzzy derivative, followed by Dubois and Prade [3], who defined and used the extension principle in their approach. Other fuzzy derivative concepts were proposed by Puri and Ralescu [4] and Goetschel and Voxman [5] as an extension of the Hukuhara derivative of multivalued functions. The

*For correspondence
FDEs and fuzzy initial value problems (FIVPs) are studied by Kaleva $[6,7]$ and Seikkala [8].

Bede [9] described the exact solutions of FDEs in his note in an excellent way. Buckley and Feuring [10] applied two analytical methods for solving $n$th order linear differential equations with fuzzy initial conditions. In the first method, they simply fuzzified the crisp solution to obtain a fuzzy function and then checked whether it satisfies the differential equation or not. And the second method was just the reverse of the first method. Ahmada et al [11] studied analytical and numerical solutions of FDEs based on the extension principle. Oregan et al [12] obtained the exact solution of fuzzy first-order boundary value problems (BVPs). In all the above-cited papers they have converted the FDEs to coupled or uncoupled system of differential equations depending on the sign of the coefficients. Very recently, a new analytical method has been developed by Tapaswini and Chakraverty [13] based on fuzzy centre, where only two crisp uncoupled differential equations are required to solve with respect to sign of the coefficients.

All fuzzy initial value problems (FIVPs) or fuzzy boundary value problems (FBVPs) may not be solved exactly. Sometimes, it is even impossible to find their analytical solutions. Hence, various numerical methods are proposed by different authors for solving FDEs, which are discussed in the following paragraph. 
A few authors have also investigated numerical methods to obtain the solution of FBVPs. Wintner-type result for fuzzy IVPs and a superlinear-type result for FBVPs are investigated by Oregan et al [12]. A new condition has been developed by Chen et al [14] to show that the two-point FBVPs and fuzzy integral equation are equivalent. Generalized differentiability concept has been applied by Khastan and Nieto [15] to obtain the solution of two-point FBVPs. Nonhomogeneous FBVPs using collocation method has been studied by Mohammed and Fadhel [16]. Jamshidi and Avazpour [17] applied shooting method for second-order fuzzy boundary value problems (SOFBVPs) under generalized differentiability. Undetermined fuzzy coefficients method has been applied by Guo et al [18] to obtain the solution of SOFBVPs. Dahalan et al [19] implemented half-sweep alternating group explicit method to obtain the numerical solution. Optimal homotopy asymptotic method (OHAM) has been applied by Jameel and Ismail [20] to solve $n$th order two-point FBVPs. Also, some application problems have been modelled through FBVPs, e.g., Chen et al [21] solved two-point boundary value undamped uncertain dynamical systems.

It is revealed from the above literature review that various authors applied different methods to solve FDEs. In general these methods are sometimes problem dependent and less efficient for large systems. Also less work has been done in the field of FBVP. Hence, here an alternative attempt has been made by using Galerkin's method to solve FBVPs. The novelty of the method is that this method along with the $r$-cut form converts the FDE into a crisp linear system of equations, which may very easily be solved by any well-known methods. Moreover the sign of the coefficients in the FDE plays an important role. As such different signs of the coefficients may also be handled in a straightforward way by using the proposed method. The powerfulness of the procedure has been demonstrated first by a few simple (fuzzy) mathematical example problems. And finally an application problem has also been considered to show the efficacy and applicability of the method.

This paper is organized as follows. In section 2, we have given basic preliminaries related to the present investigation. Next, the proposed method has been investigated in section 3. Further, in section 4, various numerical examples along with one application problem related to heat transfer have been solved and discussed. Finally in the last section conclusions are drawn.

\section{Preliminaries}

In this section, we present some notations, definitions and preliminaries, which are used further in this paper [13, 22-24].

\section{Definition 2.1 Fuzzy number}

A fuzzy number $\widetilde{U}$ is convex normalized fuzzy set $\widetilde{U}$ of the real line $R$ such that

$$
\left\{\mu_{U}^{\sim}(x): R \rightarrow[0,1], \quad \forall x \in R\right\}
$$

where $\mu_{\tilde{U}}$ is called the membership function of the fuzzy set and it is piecewise continuous.

Definition 2.2 Triangular fuzzy number

A triangular fuzzy number $\widetilde{U}$ is a convex normalized fuzzy set $\widetilde{U}$ of the real line $R$ such that

1. There exists exactly one $x_{0} \in R$ with $\mu_{U}\left(x_{0}\right)=1\left(x_{0}\right.$ is called the mean value of $\widetilde{U})$, where $\mu_{U}$ is called the membership function of the fuzzy set.

2. $\mu_{U}^{\sim}(x)$ is piecewise continuous.

Let us consider an arbitrary triangular fuzzy number $\widetilde{U}=(a, b, c)$. The membership function $\mu_{\widetilde{U}}$ of $\widetilde{U}$ will be defined as follows:

$$
\mu_{U}(x)=\left\{\begin{array}{ll}
0, & x \leq a \\
\frac{x-a}{b-a}, & a \leq x \leq b \\
\frac{c-x}{c-b}, & b \leq x \leq c \\
0, & x \geq c
\end{array} .\right.
$$

The triangular fuzzy number $\widetilde{U}=(a, b, c)$ can be represented with an ordered pair of functions through $r$-cut approach, viz. $[\underline{u}(r), \bar{u}(r)]=[(b-a) r+a,-(c-b) r+c]$, where $r \in[0,1]$.

It may be noted that the lower and upper bounds of the fuzzy numbers satisfy the following requirements:

i. $\underline{u}(r)$ is a bounded left continuous non-decreasing function over $[0,1]$.

ii. $\bar{u}(r)$ is a bounded right continuous non-increasing function over $[0,1]$.

iii. $\underline{u}(\alpha) \leq \bar{u}(\alpha), 0 \leq \alpha \leq 1$

\section{Definition 2.3 Fuzzy arithmetic}

For any two arbitrary fuzzy numbers $\widetilde{x}=[\underline{x}(r), \bar{x}(r)]$, $\tilde{y}=[y(r), \bar{y}(r)]$ and scalar $\mathrm{k}$, the fuzzy arithmetic is defined as follows:

i. $\tilde{x}=\tilde{y}$ if and only if $\underline{x}(r)=\underline{y}(r)$ and $\bar{x}(r)=\bar{y}(r)$

ii. $\widetilde{x}+\widetilde{y}=[\underline{x}(r)+\underline{y}(r), \bar{x}(r)+\bar{y}(r)]$

iii. $\tilde{x} \times \tilde{y}=\left[\begin{array}{r}\min (\underline{x}(r) \times \underline{y}(r), \underline{x}(r) \times \bar{y}(r), \bar{x}(r) \times \underline{y}(r), \\ \bar{x}(r) \times \bar{y}(r)), \\ \max (\underline{x}(r) \times \underline{y}(r), \underline{x}(r) \times \bar{y}(r), \bar{x}(r) \times \underline{y}(r), \\ \bar{x}(r) \times \bar{y}(r))\end{array}\right]$

iv. $k \widetilde{x}=\left\{\begin{array}{ll}{[k \bar{x}(r), k \underline{x}(r)],} & k<0 \\ {[k \underline{x}(r), k \bar{x}(r)],} & k \geq 0\end{array}\right.$.

Definition 2.4 $[15,25] \quad$ Let $F:(a, b) \rightarrow R_{F} \quad$ and $t_{0}=(a, b) . X$ is called differentiable at $t_{0}$ if there exists $F^{\prime}\left(t_{0}\right) \in R_{F}$ such that 
(1) for all $h>0$ sufficiently close to 0 , the Hukuhara difference $F\left(t_{0}+h\right) \Theta F\left(t_{0}\right)$ and $F\left(t_{0}\right) \Theta F\left(t_{0}-h\right)$ exist and (in metric $D$ )

$$
\lim _{h \rightarrow 0^{+}} \frac{F\left(t_{0}+h\right) \Theta F\left(t_{0}\right)}{h}=\lim _{h \rightarrow 0^{+}} \frac{F\left(t_{0}\right) \Theta F\left(t_{0}-h\right)}{h}=F^{\prime}\left(t_{0}\right),
$$

or

(2) for all $h>0$ sufficiently close to 0 , the Hukuhara difference $F\left(t_{0}\right) \Theta F\left(t_{0}+h\right)$ and $F\left(t_{0}-h\right) \Theta F\left(t_{0}\right)$ exist and (in metric $D$ )

$$
\lim _{h \rightarrow 0^{+}} \frac{F\left(t_{0}\right) \Theta F\left(t_{0}+h\right)}{-h}=\lim _{h \rightarrow 0^{+}} \frac{F\left(t_{0}-h\right) \Theta F\left(t_{0}\right)}{-h}=F^{\prime}\left(t_{0}\right) .
$$

Chalco-Cano and Roman-Flores [25] used Definition 2.4 to obtain the following results.

\section{Proposed method}

In this section, we propose a new technique based on Galerkin type to solve $n$th order FDE. Accordingly let us consider the general form of the problem as

$$
\begin{aligned}
& \widetilde{y}^{(n)}(t ; r)+a_{n-1}(t) \widetilde{y}^{(n-1)}(t ; r)+\cdots+a_{1}(t) \widetilde{y}^{\prime}(t ; r) \\
& \quad+a_{0}(t) \widetilde{y}(t ; r)=\widetilde{g}(t ; r),
\end{aligned}
$$

where $a_{i}(t), 0 \leq i \leq n-1$, is continuous on some interval $I$, subject to fuzzy boundary conditions

$$
\widetilde{y}(a ; r)=[\underline{\beta}(r), \bar{\beta}(r)], \widetilde{y}(b ; r)=[\underline{\gamma}(r), \bar{\gamma}(r)]
$$

and $\widetilde{y}(t ; r)$ is the solution to be determined.

Now an approximate solution is assumed involving the unknown fuzzy constants $\widetilde{c}_{i}$, for $i=1,2,3, \ldots, n$ as

$$
\widetilde{y}(t ; r)=\widetilde{\phi}_{0}(t ; r)+f \sum_{i=1}^{n} \widetilde{c}_{i} \Psi_{i}(t)
$$

where $\widetilde{\phi}_{0}(t ; r)=[M \underline{\beta}(r)+N \underline{\gamma}(r), M \bar{\beta}(r)+N \bar{\gamma}(r)], \quad M=$ $\frac{b-t}{b-a}, N=\frac{t-a}{b-a} f=(t-a)(b-t)$, and $\Psi_{i}(t)=t^{i-1}$.

Now one may write Eq. (2) as

$$
\widetilde{y}(t ; r)=\widetilde{\phi}_{0}(t ; r)+\sum_{i=1}^{n} \widetilde{c}_{i} \phi_{i}(t)
$$

where $\phi_{i}(t)=f \Psi_{i}(t)$.

Here it can be clearly seen that $\tilde{y}(t ; r)$ satisfies the given boundary conditions. Next, by substituting Eq. (3) in Eq. (1), the residual $\widetilde{R}\left(t ; r, \widetilde{c}_{1}, \widetilde{c}_{2}, \ldots, \widetilde{c}_{n}\right)$ may be obtained as

$$
\begin{aligned}
& \widetilde{R}\left(t ; r, \widetilde{c}_{1}, \widetilde{c}_{2}, \ldots, \widetilde{c}_{n}\right)=\left(\widetilde{\phi}_{0}^{(n)}(t ; r)+\sum_{i=1}^{n} \widetilde{c}_{i} \phi_{i}^{(n)}(t)\right) \\
& \quad+a_{n-1}(t)\left(\widetilde{\phi}_{0}^{(n-1)}(t ; r)+\sum_{i=1}^{n} \widetilde{c}_{i} \phi_{i}^{(n-1)}(t)\right)+\cdots \\
& \quad+a_{1}(t)\left(\widetilde{\phi}_{0}^{\prime}(t ; r)+\sum_{i=1}^{n} \widetilde{c}_{i} \phi_{i}^{\prime}(t)\right) \\
& \quad+a_{0}(t)\left(\widetilde{\phi}_{0}(t ; r)+\sum_{i=1}^{n} \widetilde{c}_{i} \phi_{i}(t)\right)-\widetilde{g}(t ; r)
\end{aligned}
$$

The residual $\widetilde{R}\left(t ; r, \widetilde{c}_{1}, \widetilde{c}_{2}, \ldots, \widetilde{c}_{n}\right)$ is then orthogonalized with the functions $\phi_{j}$ for $j=1,2, \ldots, n$. This gives

$$
\int_{a}^{b} \widetilde{R}\left(t ; r, \widetilde{c}_{1}, \widetilde{c}_{2}, \ldots, \widetilde{c}_{n}\right) \phi_{j}(t) d t=0
$$

Solving the above system (5) one may obtain the fuzzy constants $\widetilde{c}_{i}$. Next, substituting the value of these constants in Eq. (3) one may have the approximate solution of the $n$th order FBVP. To compare the results of proposed method we have also applied the method of Bede [9] to find the exact solution. Now three cases as below may arise:

Case 1 When the coefficients $a_{n-1}(t), a_{n-2}(t), \ldots$, $a_{1}(t), a_{0}(t)$ are all positive.

From Eq. (1) we have

$$
\begin{aligned}
& \underline{y}^{(n)}(t ; r)+a_{n-1}(t) \underline{y}^{(n-1)}(t ; r)+\cdots+a_{1}(t) \underline{y}^{\prime}(t ; r) \\
& \quad+a_{0}(t) \underline{y}(t ; r)=\underline{g}(t ; r), \\
& \bar{y}^{(n)}(t ; r)+a_{n-1}(t) \bar{y}^{(n-1)}(t ; r)+\cdots+a_{1}(t) \bar{y}^{\prime}(t ; r) \\
& \quad+a_{0}(t) \bar{y}(t ; r)=\vec{g}(t ; r) .
\end{aligned}
$$

In view of Eqs. (6) and (7), (by using Eq. (3)) we have the residues $\underline{R}\left(t ; r, \underline{c}_{1}, \underline{c}_{2}, \ldots, \underline{c}_{n}\right)$ and $\vec{R}\left(t ; r, \vec{c}_{1}, \vec{c}_{2}, \ldots, \vec{c}_{n}\right)$, respectively, as

$$
\begin{aligned}
& \underline{R}\left(t ; r, \underline{c}_{1}, \underline{c}_{2}, \ldots, \underline{c}_{n}\right)=\left(\underline{\phi}_{0}^{(n)}(t ; r)+\sum_{i=1}^{n} \underline{c}_{i} \phi_{i}^{(n)}(t)\right) \\
& \quad+a_{n-1}(t)\left(\underline{\phi}_{0}^{(n-1)}(t ; r)+\sum_{i=1}^{n} \underline{c}_{i} \phi_{i}^{(n-1)}(t)\right)+\cdots \\
& \quad+a_{1}(t)\left(\underline{\phi}_{0}^{\prime}(t ; r)+\sum_{i=1}^{n} \underline{c}_{i} \phi_{i}^{\prime}(t)\right) \\
& \quad+a_{0}(t)\left(\underline{\phi}_{0}(t ; r)+\sum_{i=1}^{n} \underline{c}_{i} \phi_{i}(t)\right)-\underline{g}(t ; r)
\end{aligned}
$$

and 


$$
\begin{aligned}
& \vec{R}\left(t ; r, \vec{c}_{1}, \vec{c}_{2}, \ldots, \vec{c}_{n}\right)=\left(\vec{\phi}_{0}^{(n)}(t ; r)+\sum_{i=1}^{n} \bar{c}_{i} \phi_{i}^{(n)}(t)\right) \\
& +a_{n-1}(t)\left(\vec{\phi}_{0}^{(n-1)}(t ; r)+\sum_{i=1}^{n} \bar{c}_{i} \phi_{i}^{(n-1)}(t)\right)+\cdots \\
& \quad+a_{1}(t)\left(\vec{\phi}_{0}^{\prime}(t ; r)+\sum_{i=1}^{n} \bar{c}_{i} \phi_{i}^{\prime}(t)\right) \\
& \quad+a_{0}(t)\left(\vec{\phi}_{0}(t ; r)+\sum_{i=1}^{n} \bar{c}_{i} \phi_{i}(t)\right)-\bar{g}(t ; r)
\end{aligned}
$$

The residual $\widetilde{R}\left(t ; r, \widetilde{\mathrm{c}}_{1}, \widetilde{\mathrm{c}}_{2}, \ldots, \widetilde{\mathrm{c}}_{n}\right)=\left[\underline{R}\left(t ; r, \underline{c}_{1}, \underline{c}_{2}, \ldots\right.\right.$, $\left.\left.\underline{c}_{n}\right), \vec{R}\left(t ; r, \vec{c}_{1}, \vec{c}_{2}, \ldots, \vec{c}_{n}\right)\right]$ is then orthogonalized with the functions $\phi_{j}$ for $j=1,2, \ldots, n$. This gives

$$
\begin{aligned}
& \int_{a}^{b}\left\{\left(\underline{\phi}_{-}^{(n)}(t ; r)+\sum_{i=1}^{n} \underline{c}_{i} \phi_{i}^{(n)}(t)\right)\right. \\
& +a_{n-1}(t)\left(\underline{\phi}_{0}^{(n-1)}(t ; r)+\sum_{i=1}^{n} \underline{c}_{i} \phi_{i}^{(n-1)}(t)\right)+\cdots \\
& \left.+a_{0}(t)\left(\underline{\phi}_{0}(t ; r)+\sum_{i=1}^{n} \underline{c}_{i} \phi_{i}(t)\right)-\underline{g}(t ; r)\right\} \phi_{j} d t \\
& \int_{a}^{b}\left\{\left(\vec{\phi}_{0}^{(n)}(t ; r)+\sum_{i=1}^{n} \bar{c}_{i} \phi_{i}^{(n)}(t)\right)\right. \\
& +a_{n-1}(t)\left(\vec{\phi}_{0}^{(n-1)}(t ; r)+\sum_{i=1}^{n} \bar{c}_{i} \phi_{i}^{(n-1)}(t)\right)+\cdots \\
& \left.\quad+a_{0}(t)\left(\vec{\phi}_{0}(t ; r)+\sum_{i=1}^{n} \bar{c}_{i} \phi_{i}(t)\right)-\bar{g}(t ; r)\right\} \phi_{j} d t
\end{aligned}
$$

We may write Eqs. (10) and (11) as

$$
\begin{aligned}
& \int_{a}^{b} \sum_{i=1}^{n} \underline{c}_{i} \phi_{j}\left\{\phi_{i}^{(n)}(t)+\cdots+a_{0}(t) \phi_{i}(t)\right\} d t \\
& \quad=\int_{a}^{b} \phi_{j}\left\{\underline{g}(t ; r)-\left(\underline{\phi}_{0}^{(n)}(t)+\cdots+a_{0}(t) \underline{\phi}_{0}(t)\right)\right\} d t \\
& \int_{a}^{b} \sum_{i=1}^{n} \bar{c}_{i} \phi_{j}\left\{\phi_{i}^{(n)}(t)+\cdots+a_{0}(t) \phi_{i}(t)\right\} d t \\
& \quad=\int_{a}^{b} \phi_{j}\left\{\bar{g}(t ; r)-\left(\bar{\phi}_{0}^{(n)}(t)+\cdots+a_{0}(t) \bar{\phi}_{0}(t)\right)\right\} d t
\end{aligned}
$$

Let us assume

$$
\begin{aligned}
& \int_{a}^{b} \phi_{j}\left\{\phi_{i}^{(n)}(t)+a_{n-1}(t) \phi_{i}^{(n-1)}(t)+\cdots+a_{0}(t) \phi_{i}(t)\right\} d t \\
& \quad=\alpha_{j i}, \\
& \int_{a}^{b} \phi_{j}\left\{\underline{g}(t ; r)-\left(\underline{\phi}_{0}^{(n)}(t)+a_{n-1}(t) \underline{\phi}_{0}^{(n-1)}(t)+\cdots+a_{0}(t) \underline{\phi}_{0}(t)\right)\right\} \\
& \quad=f_{j}(t ; r)
\end{aligned}
$$

and

$$
\begin{aligned}
& \int_{a}^{b} \phi_{j}\left\{\bar{g}(t ; r)-\left(\bar{\phi}_{0}^{(n)}(t)+a_{n-1}(t) \bar{\phi}_{0}^{(n-1)}(t)+\cdots+a_{0}(t) \bar{\phi}_{0}(t)\right)\right\} d t \\
& \quad=\bar{f}_{j}(t ; r)
\end{aligned}
$$

Then the following system is obtained:

$$
\begin{aligned}
\sum_{i=1}^{n} \alpha_{j i} c_{i} & =f_{-j}(t ; r) \\
\sum_{i=1}^{n} \alpha_{j i} \bar{c}_{i} & =\bar{f}_{j}(t ; r)
\end{aligned} \quad \text { for } \quad j=1,2, \ldots, n .
$$

The above system (14) can be written in the form of a linear system of equations $A X=B$ as follows:

$$
A=\left[\begin{array}{ll}
A_{1} & A_{2} \\
A_{2} & A_{1}
\end{array}\right]_{2 n \times 2 n}
$$

where

$$
\begin{aligned}
A_{1} & =\left[\begin{array}{ccccc}
\alpha_{11} & \alpha_{12} & \alpha_{13} & \cdots & \alpha_{1 n} \\
\alpha_{21} & \alpha_{22} & \alpha_{23} & \cdots & \alpha_{2 n} \\
\alpha_{31} & \alpha_{32} & \alpha_{33} & \cdots & \alpha_{3 n} \\
\vdots & \vdots & \vdots & \cdots & \vdots \\
\alpha_{n 1} & \alpha_{n 2} & \alpha_{3 n} & \cdots & \alpha_{n n}
\end{array}\right]_{n \times n}, \\
A_{2} & =\left[\begin{array}{ccccc}
0 & 0 & 0 & \cdots & 0 \\
0 & 0 & 0 & \cdots & 0 \\
0 & 0 & 0 & \cdots & \vdots \\
\vdots & \vdots & \vdots & \cdots & 0 \\
0 & 0 & 0 & \cdots & 0
\end{array}\right]_{n \times n} \\
X & =\left[c_{1}, \underline{c}_{2}, \ldots, \underline{c}_{n}, \bar{c}_{1}, \bar{c}_{2}, \ldots, \bar{c}_{n}\right]
\end{aligned}
$$

and $\quad B=\left[f_{-1}(t ; r), f_{-2}(t ; r), \ldots, f_{-n}(t ; r), \bar{f}_{1}(t ; r), \bar{f}_{2}(t ; r), \ldots\right.$, $\left.\bar{f}_{n}(t ; r)\right]$. The fuzzy constants $\widetilde{c}_{i}$ for $i=1,2, \ldots, n$ are obtained by solving the above system of equations (Eq. (14)). Substituting these constants in Eq. (3) we get the approximate solution of the FBVP.

Case 2 When the coefficients $a_{n-1}(t), a_{n-2}(t), \ldots$, $a_{1}(t), a_{0}(t)$ are all negative.

From Eq. (1) we have 


$$
\begin{gathered}
\underline{y}^{(n)}(t ; r)+a_{n-1}(t) \bar{y}^{(n-1)}(t ; r)+\cdots \\
+a_{1}(t) \bar{y}^{\prime}(t ; r)+a_{0}(t) \bar{y}(t ; r)=\underline{g}(t ; r), \\
\bar{y}^{(n)}(t ; r)+a_{n-1}(t) \underline{y}^{(n-1)}(t ; r)+\cdots+a_{1}(t) \underline{y}^{\prime}(t ; r) \\
+a_{0}(t) \underline{y}(t ; r)=\vec{g}(t ; r) .
\end{gathered}
$$

In view of Eqs. (15) and (16), (by using Eq. (3)) we may have the residues $\underline{R}\left(t ; r, \underline{c}_{1}, \underline{c}_{2}, \ldots, \underline{c}_{n}\right)$ and $\vec{R}\left(t ; r, \vec{c}_{1}\right.$, $\left.\vec{c}_{2}, \ldots, \vec{c}_{n}\right)$, respectively, as

$$
\begin{aligned}
& \underline{R}\left(t ; r, \underline{c}_{1}, \underline{c}_{2}, \ldots, \underline{c}_{n}\right)=\left(\underline{\phi}_{0}^{(n)}(t ; r)+\sum_{i=1}^{n} \underline{c}_{i} \phi_{i}^{(n)}(t)\right) \\
& \quad+a_{n-1}(t)\left(\bar{\phi}_{0}^{(n-1)}(t ; r)+\sum_{i=1}^{n} \bar{c}_{i} \phi_{i}^{(n-1)}(t)\right)+\cdots \\
& \quad+a_{1}(t)\left(\bar{\phi}_{0}^{\prime}(t ; r)+\sum_{i=1}^{n} \bar{c}_{i} \phi_{i}^{\prime}(t)\right) \\
& \quad+a_{0}(t)\left(\bar{\phi}_{0}(t ; r)+\sum_{i=1}^{n} \bar{c}_{i} \phi_{i}(t)\right)-\underline{g}(t ; r)
\end{aligned}
$$

and

$$
\begin{aligned}
& \vec{R}\left(t ; r, \vec{c}_{1}, \vec{c}_{2}, \ldots, \vec{c}_{n}\right)=\left(\vec{\phi}_{0}^{(n)}(t ; r)+\sum_{i=1}^{n} \bar{c}_{i} \phi_{i}^{(n)}(t)\right) \\
& +a_{n-1}(t)\left(\underline{\phi}_{0}^{(n-1)}(t ; r)+\sum_{i=1}^{n} \underline{c}_{i} \phi_{i}^{(n-1)}(t)\right) \\
& \quad+a_{1}(t)\left(\underline{\phi}_{0}^{\prime}(t ; r)+\sum_{i=1}^{n} \underline{c}_{i} \phi_{i}^{\prime}(t)\right) \\
& \quad+a_{0}(t)\left(\underline{\phi}_{0}(t ; r)+\sum_{i=1}^{n} \underline{c}_{i} \phi_{i}(t)\right)-\bar{g}(t ; r) .
\end{aligned}
$$

The residual $\widetilde{R}\left(t ; r, \widetilde{\mathrm{c}}_{1}, \widetilde{\mathrm{c}}_{2}, \ldots, \widetilde{\mathrm{c}}_{n}\right)=\left[\underline{R}\left(t ; r, \underline{c}_{1}, \underline{c}_{2}, \ldots\right.\right.$, $\left.\left.\underline{c}_{n}\right), \vec{R}\left(t ; r, \vec{c}_{1}, \vec{c}_{2}, \ldots, \vec{c}_{n}\right)\right]$ is then orthogonalized with the functions $\phi_{j}$ for $j=1,2, \ldots, n$. This gives

$$
\begin{gathered}
\int_{a}^{b} \underline{R}\left(t ; r, \underline{c}_{1}, \underline{c}_{2}, \ldots, \underline{c}_{n}\right) \phi_{j} d t \\
\int_{a}^{b} \vec{R}\left(t ; r, \vec{c}_{1}, \vec{c}_{2}, \ldots, \vec{c}_{n}\right) \phi_{j} d t .
\end{gathered}
$$

We may write Eqs. (19) and (20) as

$$
\begin{aligned}
& \int_{a}^{b} \phi_{j}\left\{\sum_{i=1}^{n} \underline{c}_{i} \phi_{i}^{(n)}(t)+a_{n-1}(t) \sum_{i=1}^{n} \bar{c}_{i} \phi_{i}^{(n-1)}(t)+\cdots\right. \\
& \left.+a_{0}(t) \sum_{i=1}^{n} \bar{c}_{i} \phi_{i}(t)\right\} d t \\
& =\int_{a}^{b} \phi_{j}\left\{\underline{g}(t ; r)-\left(\underline{\phi}_{0}^{(n)}(t)+a_{n-1}(t) \bar{\phi}_{0}^{(n-1)}(t)+\cdots\right.\right. \\
& \left.\left.\quad+a_{0}(t) \bar{\phi}_{0}(t)\right)\right\} d t \\
& \int_{a}^{b} \phi_{j}\left\{\sum_{i=1}^{n} \bar{c}_{i} \phi_{i}^{(n)}(t)+a_{n-1}(t) \sum_{i=1}^{n} \underline{c}_{i} \phi_{i}^{(n-1)}(t)+\cdots\right. \\
& \left.+a_{0}(t) \sum_{i=1}^{n} \underline{c}_{i} \phi_{i}(t)\right\} d t \\
& =\int_{a}^{b} \phi_{j}\left\{\bar{g}_{(t ; r)-\left(\bar{\phi}_{0}^{(n)}(t)+a_{n-1}(t) \underline{\phi}_{0}^{(n-1)}(t)+\cdots\right.}\right. \\
& \left.\left.\quad+a_{0}(t) \underline{\phi}_{0}(t)\right)\right\} d t .
\end{aligned}
$$

Again by taking

$$
\begin{gathered}
\int_{a}^{b} \phi_{j} \phi_{i}^{(n)}(t) d t=\eta_{i, i} \\
\int_{a}^{b} \phi_{j}\left\{a_{n-1}(t) \phi_{i}^{(n-1)}(t)+\cdots+a_{0}(t) \phi_{i}(t)\right\} d t=\delta_{i, i}, \\
\int_{a}^{b} \phi_{j}\left\{\underline{g}(t ; r)-\left(\underline{\phi}_{0}^{(n)}(t)+a_{n-1}(t) \bar{\phi}_{0}^{(n-1)}(t)+\cdots\right.\right. \\
\left.\left.+a_{0}(t) \bar{\phi}_{0}(t)\right)\right\}=f_{-j}(t ; r)
\end{gathered}
$$

and

$$
\begin{aligned}
& \int_{a}^{b} \phi_{i}\left\{\bar{g}(t ; r)-\left(\bar{\phi}_{0}^{(n)}(t)+a_{n-1}(t) \underline{\phi}_{0}^{(n-1)}(t)+\cdots\right.\right. \\
& \left.\left.\quad+a_{0}(t) \underline{\phi}_{0}(t)\right)\right\}=\bar{f}_{j}(t ; r)
\end{aligned}
$$

the following system is obtained: 


$$
\left(\sum_{i=1}^{n} \eta_{j i} \underline{c}_{i}+\sum_{i=1}^{n} \delta_{j i} \bar{c}_{i}\right)=f_{-j}(t ; r) .
$$

The above system (23) may again be represented as a system of linear equation $A X=B$ such as

$$
A=\left[\begin{array}{ll}
A_{1} & A_{2} \\
A_{2} & A_{1}
\end{array}\right]_{2 n \times 2 n}
$$

where

$$
\begin{gathered}
A_{1}=\left[\begin{array}{ccccc}
\eta_{11} & \eta_{12} & \eta_{13} & \cdots & \eta_{1 n} \\
\eta_{21} & \eta_{22} & \eta_{23} & \cdots & \eta_{2 n} \\
\eta_{31} & \eta_{32} & \eta_{33} & \cdots & \eta_{3 n} \\
\vdots & \vdots & \vdots & \cdots & \vdots \\
\eta_{n 1} & \eta_{n 2} & \eta_{3 n} & \cdots & \eta_{n n}
\end{array}\right]_{n \times n}, \\
A_{2}=\left[\begin{array}{ccccc}
\delta_{11} & \delta_{12} & \delta_{13} & \cdots & \delta_{1 n} \\
\delta_{21} & \delta_{22} & \delta_{23} & \cdots & \delta_{2 n} \\
\delta_{31} & \delta_{32} & \delta_{33} & \cdots & \delta_{3 n} \\
\vdots & \vdots & \vdots & \cdots & \vdots \\
\delta_{n 1} & \delta_{n 2} & \delta_{3 n} & \cdots & \delta_{n n}
\end{array}\right]_{n \times n}, \\
X=\left[\begin{array}{lll}
\underline{c}_{1}, \underline{c}_{2}, \ldots, \underline{c}_{n}, \bar{c}_{1}, \bar{c}_{2}, \ldots, \bar{c}_{n}
\end{array}\right]
\end{gathered}
$$

and $B=\left[f_{1}(t ; r), f_{-2}(t ; r), \ldots, f_{-n}(t ; r), \bar{f}_{1}(t ; r), \bar{f}_{2}(t ; r), \ldots, \bar{f}_{n}\right.$ $(t ; r)]$. The fuzzy constants $\widetilde{c}_{i}$ for $i=1,2, \ldots, n$ are obtained by solving the above system of equations Eq. (23). Substitute these constants in Eq. (3) to get the approximate solution of Eqs. (15) and (16) for this case.

Case 3 When the coefficients $a_{n-m-1}(t), a_{n-m-2}(t)$, $\ldots, a_{1}(t), a_{0}(t)$ for $n \geq m$ are negative. From Eq. (1) we have

$$
\begin{aligned}
& \underline{y}^{(n)}(t ; r)+a_{n-1}(t) \underline{y}^{(n-1)}(t ; r)+\cdots \\
& \quad+a_{n-m}(t) \underline{y}^{(n-m)}(t ; r)+a_{n-m-1}(t) \bar{y}^{(n-m-1)}(t ; r)+\cdots \\
& \quad+a_{0}(t) \bar{y}(t ; r)=\underline{g}(t ; r), \\
& \quad \bar{y}^{(n)}(t ; r)+a_{n-1}(t) \bar{y}^{(n-1)}(t ; r)+\cdots+a_{n-m}(t) \bar{y}^{(n-m)}(t ; r) \\
& \quad+a_{n-m-1}(t) \underline{y}^{(n-m-1)}(t ; r)+\cdots \\
& \quad+a_{0}(t) \underline{y}(t ; r)=\bar{g}(t ; r) .
\end{aligned}
$$

We may have the residues $\underline{R}\left(t ; r, \underline{c}_{1}, \underline{c}_{2}, \ldots, \underline{c}_{n}\right)$ and $\vec{R}\left(t ; r, \vec{c}_{1}, \vec{c}_{2}, \ldots, \vec{c}_{n}\right)$, respectively, as

$$
\begin{aligned}
& \underline{R}\left(t ; r, \underline{c}_{1}, \underline{c}_{2}, \ldots, \underline{c}_{n}\right)=\left(\underline{\phi}_{-}^{(n)}(t ; r)+\sum_{i=1}^{n} \underline{c}_{i} \phi_{i}^{(n)}(t)\right) \\
& +a_{n-1}(t)\left(\underline{\phi}_{0}^{(n-1)}(t ; r)+\sum_{i=1}^{n} \underline{c}_{i} \phi_{i}^{(n-1)}(t)\right)+\cdots \\
& +a_{n-m}(t)\left(\underline{\phi}^{(n-m)}(t ; r)+\sum_{i=1}^{n} \underline{c}_{i} \phi_{i}^{(n-m)}(t)\right) \\
& +a_{n-m-1}(t)\left(\bar{\phi}_{0}^{(n-m-1)}(t ; r)+\sum_{i=1}^{n} \bar{c}_{i} \phi_{i}^{(n-m-1)}(t)\right)+\cdots \\
& +a_{0}(t)\left(\bar{\phi}_{0}(t ; r)+\sum_{i=0}^{n} \bar{c}_{i} \phi_{i}(t)\right)-\underline{g}(t ; r) \\
& \vec{R}\left(t ; r, \vec{c}_{1}, \vec{c}_{2}, \ldots, \vec{c}_{n}\right)=\left(\bar{\phi}_{0}^{(n)}(t ; r)+\sum_{i=1}^{n} \bar{c}_{i} \phi_{i}^{(n)}(t)\right) \\
& +a_{n-1}(t)\left(\bar{\phi}_{0}^{(n-1)}(t ; r)+\sum_{i=1}^{n} \bar{c}_{i} \phi_{i}^{(n-1)}(t)\right)+\cdots \\
& +a_{n-m}(t)\left(\bar{\phi}_{0}^{(n-m)}(t ; r)+\sum_{i=1}^{n} \bar{c}_{i} \phi_{i}^{(n-m)}(t)\right) \\
& +a_{n-m-1}(t)\left(\underline{\phi}_{0}^{(n-m-1)}(t ; r)+\sum_{i=1}^{n} \underline{c}_{i} \phi_{i}^{(n-m-1)}(t)\right)+\cdots \\
& +a_{0}(t)\left(\underline{\phi}_{0}(t ; r)+\sum_{i=0}^{n} \underline{c}_{i} \phi_{i}(t)\right)-\bar{g}(t ; r)
\end{aligned}
$$

The residual $\widetilde{R}\left(t ; r, \widetilde{\mathbf{c}}_{1}, \widetilde{\mathbf{c}}_{2}, \ldots, \widetilde{\mathbf{c}}_{n}\right)=\left[\underline{R}\left(t ; r, \underline{c}_{1}, \underline{c}_{2}, \ldots\right.\right.$, $\left.\left.\underline{c}_{n}\right), \vec{R}\left(t ; r, \vec{c}_{1}, \vec{c}_{2}, \ldots, \vec{c}_{n}\right)\right]$ is then orthogonalized with the functions $\phi_{j}$ for $j=1,2, \ldots, n$ as done in previous cases. Next we get

$$
\int_{a}^{b} \underline{R}\left(t ; r, \underline{c}_{1}, \underline{c}_{2}, \ldots, \underline{c}_{n}\right) \phi_{j} d t
$$

and

$$
\int_{a}^{b} \vec{R}\left(t ; r, \vec{c}_{1}, \vec{c}_{2}, \ldots, \vec{c}_{n}\right) \phi_{j} d t
$$

Eqs. (28) and (29) are now written as 


$$
\begin{aligned}
\int_{a}^{b} \phi_{j} & \left\{\sum_{i=1}^{n} \underline{c}_{i} \phi_{i}^{(n)}(t)+\cdots+a_{n-m}(t) \sum_{i=1}^{n} \underline{c}_{i} \phi_{i}^{(n-m)}(t)\right. \\
+ & \left.a_{n-m-1}(t) \sum_{i=1}^{n} \bar{c}_{i} \phi_{i}^{(n-m-1)}(t)+a_{0}(t) \sum_{i=1}^{n} \bar{c}_{i} \phi_{i}(t)\right\} d t \\
= & \int_{a}^{b} \phi_{j}\left\{\underline{g}(t ; r)-\left(\underline{\phi}_{0}^{(n)}(t)+a_{n-1}(t) \underline{\phi}^{(n-1)}(t)+\cdots\right.\right. \\
& +a_{n-m}(t) \underline{\phi}^{(n-m)}(t)+a_{n-m-1}(t) \bar{\phi}_{0}^{(n-m-1)}(t)+\cdots \\
& \left.\left.+a_{0}(t) \bar{\phi}_{0}(t)\right)\right\} d t \\
\int_{a}^{b} & \oint_{j}\left\{\sum_{i=1}^{n} \bar{c}_{i} \phi_{i}^{(n)}(t)+\cdots+a_{n-m}(t) \sum_{i=1}^{n} \bar{c}_{i} \phi_{i}^{(n-m)}(t)\right. \\
+ & \left.a_{n-m-1}(t) \sum_{i=1}^{n} \underline{c}_{i} \phi_{i}^{(n-m-1)}(t)+a_{0}(t) \sum_{i=1}^{n} \underline{c}_{i} \phi_{i}(t)\right\} d t \\
+ & \left.\left.a_{n-m}(t) \underline{\phi}_{0}(t)\right)\right\} d t \\
= & \int_{b} \phi_{j}\left\{\bar{g}(t ; r)-\left(\bar{\phi}_{0}^{(n)}(t)+a_{n-1}(t) \bar{\phi}_{0}^{(n-1)}(t)+\cdots\right.\right.
\end{aligned}
$$

Again by assuming

$$
\begin{gathered}
\int_{a}^{b} \phi_{j}\left\{\phi_{i}^{(n)}+a_{n-1} \phi_{i}^{(n-1)}(t)+\cdots+a_{n-m} \phi_{i}^{(n-m)}(t)\right\} d t=\xi_{j i} \\
\int_{a}^{b} \phi_{j}\left\{a_{n-m-1}(t) \phi_{i}^{(n-m-1)}(t)+\cdots+a_{1}(t) \phi_{i}^{\prime}(t)+a_{0} \phi_{i}(t)\right\} d t \\
=\mu_{j i}, \\
\quad b \quad \phi_{j}\left\{\underline{g}(t ; r)-\left(\underline{\phi}_{0}^{(n)}(t)+a_{n-1}(t) \underline{\phi}_{0}^{(n-1)}(t)+\cdots\right.\right. \\
\quad+a_{n-m} \underline{\phi}_{0}^{(n-m)}(t)+a_{n-m-1} \bar{\phi}_{0}^{(n-m-1)}(t)+\cdots \\
\left.\left.+a_{0}(t) \bar{\phi}_{0}(t)\right)\right\}=f_{j}(t ; r)
\end{gathered}
$$

where

the following system is

$$
\left(\sum_{i=1}^{n} \xi_{j i} \underline{c}_{i}+\sum_{i=1}^{n} \mu_{j i} \bar{c}_{i}\right)=f_{j}(t ; r) \quad \text { for } j=1,2, \ldots, n \text {. }
$$

Eq. (32) is written finally in the form of $A X=B$ as

$$
A=\left[\begin{array}{ll}
A_{1} & A_{2} \\
A_{2} & A_{1}
\end{array}\right]_{2 n \times 2 n}
$$

$$
\begin{aligned}
A_{1}= & {\left[\begin{array}{ccccc}
\xi_{11} & \xi_{12} & \xi_{13} & \cdots & \xi_{1 n} \\
\xi_{21} & \xi_{22} & \xi_{23} & \cdots & \xi_{2 n} \\
\xi_{31} & \xi_{32} & \xi_{33} & \cdots & \xi_{3 n} \\
\vdots & \vdots & \vdots & \cdots & \vdots \\
\xi_{n 1} & \xi_{n 2} & \xi_{3 n} & \cdots & \xi_{n n}
\end{array}\right]_{n \times n}, } \\
A_{2}= & {\left[\begin{array}{ccccc}
\mu_{11} & \mu_{12} & \mu_{13} & \cdots & \mu_{1 n} \\
\mu_{21} & \mu_{22} & \mu_{23} & \cdots & \mu_{2 n} \\
\mu_{31} & \mu_{32} & \mu_{33} & \cdots & \mu_{3 n} \\
\vdots & \vdots & \vdots & \cdots & \vdots \\
\mu_{n 1} & \mu_{n 2} & \mu_{3 n} & \cdots & \mu_{n n}
\end{array}\right]_{n \times n}, }
\end{aligned}
$$

$X=\left[\underline{c}_{1}, \underline{c}_{2}, \ldots, \underline{c}_{n}, \bar{c}_{1}, \bar{c}_{2}, \ldots, \bar{c}_{n}\right] \quad$ and $\quad B=\left[f_{1}(t ; r), \underline{f}_{2}\right.$ $\left.(t ; r), \ldots, f_{n}(t ; r), \bar{f}_{1}(t ; r), \bar{f}_{2}(t ; r), \ldots, \bar{f}_{n}(t ; r)\right]$. The fuzzy constants $\widetilde{c}_{i}$ for $i=1,2, \ldots, n$ are obtained by solving the above system of equations [Eq. (32)] as done previously. These constants are substituted in Eq. (3) to get the approximate solution of the BVP.

\section{Numerical implementation of the proposed method}

In the following paragraphs, example problems are solved using the proposed method with different cases and are also compared with exact solutions. We also obtain the exact solution by following the method of Bede [9].

Example 1 Let us consider the following second-order fuzzy linear differential equation with positive coefficients (Case 1):

$$
\widetilde{y}^{\prime \prime}+\widetilde{y}+t=0
$$

subject to the fuzzy boundary conditions

$$
\widetilde{y}(0)=\widetilde{y}(1)=[0.1 r-0.1,0.1-0.1 r] .
$$

The exact fuzzy solution are found, Bede [9], as 


$$
\begin{aligned}
\underline{Y}(t ; r)= & -t+(0.1 r-0.1) \cos (t) \\
& +(1.13376+0.0546302 r) \sin (t), \\
\bar{Y}(t ; r)= & -t+(0.1-0.1 r) \cos (t) \\
& +(1.24303-0.0546302 r) \sin (t) .
\end{aligned}
$$

Let the approximate solution of Eq. (33) be

$$
\widetilde{y}(t ; r)=\widetilde{\phi}_{0}(t ; r)+\sum_{i=1}^{3} \widetilde{c}_{i} \phi_{i}(t)
$$

where $\widetilde{\phi}_{0}(t ; r)=[(0.1 r-0.1),(0.1-0.1 r)]$.

Now, $\widetilde{y}(t ; r)$ can be written as

$$
\widetilde{y}(t ; r)=\widetilde{\phi}_{0}(t ; r)+t(1-t)\left(\widetilde{c}_{1}+\widetilde{c}_{2}+t^{2} \widetilde{c}_{3}\right)
$$

which satisfies the fuzzy boundary condition.

Therefore residue $\widetilde{R}$ can be written as

$$
\begin{aligned}
& \underline{R}\left(t, r, \underline{c}_{1}, \underline{c}_{2}, \underline{c}_{3}\right)=(-0.1+0.1 r)+t \\
& \quad+\left(-2+t-t^{2}\right) \underline{c}_{1}+\left(2-6 t+t^{2}-t^{3}\right) \underline{c}_{2} \\
& \quad+t\left(6-12 t+t^{2}-t^{3}\right) \underline{c}_{3}, \\
& \quad \bar{R}\left(t, r, \bar{c}_{1}, \bar{c}_{2}, \bar{c}_{3}\right)=(0.1-0.1 r)+t+\left(-2+t-t^{2}\right) \bar{c}_{1} \\
& \quad+\left(2-6 t+t^{2}-t^{3}\right) \bar{c}_{2}+t\left(6-12 t+t^{2}-t^{3}\right) \bar{c}_{3} .
\end{aligned}
$$

Here the residue $\widetilde{R}$ is orthogonalized to the functions $\phi_{1}(t), \phi_{2}(t)$ and $\phi_{3}(t)$. This gives

$$
\begin{aligned}
& \int_{0}^{1} \widetilde{\phi}_{1}(t ; r) \widetilde{R}\left(t ; r, \widetilde{c}_{1}, \widetilde{c}_{2}, \widetilde{c}_{3}\right) d t=0, \\
& \int_{0}^{1} \widetilde{\phi}_{2}(t ; r) \widetilde{R}\left(t ; r, \widetilde{c}_{1}, \widetilde{c}_{2}, \widetilde{c}_{3}\right) d t=0,
\end{aligned}
$$

and

$$
\int_{0}^{1} \widetilde{\phi}_{3}(t ; r) \widetilde{R}\left(t ; r, \widetilde{c}_{1}, \widetilde{c}_{2}, \widetilde{c}_{3}\right) d t=0 .
$$

On solving the above equations we get the following system:
Solving for $\widetilde{c}_{1}, \widetilde{c}_{2}$ and $\widetilde{c}_{3}$ from the above system, we get

$$
\widetilde{c}_{1}=[0.132778+0.054623 r, 0.242024-0.054623 r]
$$

$$
\widetilde{c}_{2}=[0.190205+0.00469417 r, 0.199594-0.00469417 r]
$$

and

$\widetilde{c}_{3}=[-0.0187767-0.00469417 r,-0.028165+0.00469417 r]$.

By substituting the values of fuzzy constants to Eq. (35), we have

$$
\begin{aligned}
\underline{y}(t ; r)= & (0.1 r-0.1)+t(t-1)(0.132778+0.054623 r \\
& +t(0.190205+0.00469417 r) \\
& \left.+t^{2}(-0.0187767-0.00469417 r)\right), \\
\bar{y}(t ; r)= & (0.1-0.1 r)+t(t-1)(0.242024-0.054623 r \\
& +t(0.199594-0.00469417 r) \\
& \left.+t^{2}(-0.028165+0.00469417 r)\right) .
\end{aligned}
$$

Hence from this one may have the final solution as $\tilde{y}(t ; r)=[y(t ; r), \tilde{y}(t ; r)]$. Now the results obtained by the proposed method are compared with the exact solution for particular value of $t$. The solution bounds are shown in tables 1 and 2. Corresponding fuzzy plots are given in figure 1 .

Example 2 Now, we take the following second-order fuzzy linear differential equation

$$
\widetilde{y}^{\prime \prime}+\widetilde{y}=t, t \in[0,1]
$$

subject to the fuzzy boundary conditions as

$$
\widetilde{y}(0)=(3+r, 5-r), \quad \widetilde{y}(1)=(r, 2-r) .
$$

Exact fuzzy solution are obtained again by following the method of Bede [9] as

$$
\begin{aligned}
& \underline{Y}(t ; r)=t+(3+r) \cos (t)-(3+r) \cot (1) \sin (t) \\
& \quad+(r-1) \operatorname{cosec}(1) \sin (t),
\end{aligned}
$$

$\left.\begin{array}{ccc}0 & 0 & 0 \\ 0 & 0 & 0 \\ 0 & 0 & 0 \\ 0.0595238 & 0.0297619 & 0.0123016 \\ 0.0297619 & 0.0218254 & 0.0130952 \\ 0.0170635 & 0.0154762 & 0.0111472\end{array}\right]\left\{\begin{array}{c}\underline{c}_{1} \\ \underline{c}_{2} \\ \underline{c}_{3} \\ \bar{c}_{1} \\ \bar{c}_{2} \\ \bar{c}_{3}\end{array}\right\}=$

$$
\left[\begin{array}{c}
0.0133333+0.00333333 r \\
0.00785714+0.00166667 r \\
0.005+0.000952381 r \\
0.02-0.00333333 r \\
0.0111905-0.00166667 r \\
0.00690476-0.000952381 r
\end{array}\right]
$$


Table 1. Fuzzy solution of Example 1 at $t=0.1$.

\begin{tabular}{|c|c|c|c|c|c|c|}
\hline$r$ & $\underline{y}(t ; r)$ & $\underline{Y}(t ; r)$ & Error & $\bar{y}(t ; r)$ & $\bar{Y}(t ; r)$ & Error \\
\hline 0 & -0.08635504166 & -0.0863127972 & $4.2244 \mathrm{e}-5$ & 0.1235531518 & 0.1235958847 & $4.2733 e-5$ \\
\hline 0.1 & -0.07585963199 & -0.07581736311 & $4.2269 \mathrm{e}-5$ & 0.1130577421 & 0.1131004506 & $4.2709 \mathrm{e}-5$ \\
\hline 0.2 & -0.06536422231 & -0.06532192901 & $4.2293 e-5$ & 0.1025623325 & 0.1026050165 & $4.2684 \mathrm{e}-5$ \\
\hline 0.3 & -0.05486881264 & -0.05482649492 & $4.2318 \mathrm{e}-5$ & 0.09206692278 & 0.09210958239 & $4.2660 \mathrm{e}-5$ \\
\hline 0.4 & -0.04437340297 & -0.04433106083 & $4.2342 \mathrm{e}-5$ & 0.08157151311 & 0.0816141483 & $4.2635 \mathrm{e}-5$ \\
\hline 0.5 & -0.03387799329 & -0.03383562673 & $4.2367 e-5$ & 0.07107610343 & 0.0711187142 & $4.2611 \mathrm{e}-5$ \\
\hline 0.6 & -0.02338258362 & -0.02334019264 & $4.2391 \mathrm{e}-5$ & 0.06058069376 & 0.06062328011 & $4.2586 \mathrm{e}-5$ \\
\hline 0.7 & -0.01288717395 & -0.01284475854 & $4.2415 e-5$ & 0.05008528409 & 0.05012784602 & $4.2562 \mathrm{e}-5$ \\
\hline 0.8 & -0.002391764276 & -0.002349324451 & $4.2440 \mathrm{e}-5$ & 0.03958987442 & 0.03963241192 & $4.2538 \mathrm{e}-5$ \\
\hline 0.9 & 0.008103645397 & 0.008146109643 & $4.2464 \mathrm{e}-5$ & 0.02909446474 & 0.02913697783 & $4.2513 e-5$ \\
\hline 1 & 0.01859905507 & 0.01864154374 & $4.2489 \mathrm{e}-5$ & 0.01859905507 & 0.01864154374 & $4.2489 \mathrm{e}-5$ \\
\hline
\end{tabular}

Table 2. Fuzzy solution of Example 1 at $t=0.01$.

\begin{tabular}{|c|c|c|c|c|c|c|}
\hline$r$ & $\underline{y}(t ; r)$ & $\underline{Y}(t ; r)$ & Error & $\bar{y}(t ; r)$ & $\bar{Y}(t ; r)$ & Error \\
\hline 0 & -0.09866668715 & -0.09865754043 & $9.1467 \mathrm{e}-6$ & 0.1024157693 & 0.1024250464 & $9.2771 \mathrm{e}-6$ \\
\hline 0.1 & -0.08861256433 & -0.08860341109 & $9.1532 \mathrm{e}-6$ & 0.09236164645 & 0.09237091708 & $9.2706 e-6$ \\
\hline 0.2 & -0.07855844151 & -0.07854928175 & $9.1598 \mathrm{e}-6$ & 0.08230752363 & 0.08231678773 & $9.2641 \mathrm{e}-6$ \\
\hline 0.3 & -0.06850431869 & -0.06849515241 & $9.1663 \mathrm{e}-6$ & 0.07225340081 & 0.07226265839 & $9.2576 e-6$ \\
\hline 0.4 & -0.05845019587 & -0.05844102306 & $9.1728 \mathrm{e}-6$ & 0.06219927799 & 0.06220852905 & $9.2511 \mathrm{e}-6$ \\
\hline 0.5 & -0.04839607305 & -0.04838689372 & $9.1793 \mathrm{e}-6$ & 0.05214515517 & 0.05215439971 & $9.2445 \mathrm{e}-6$ \\
\hline 0.6 & -0.03834195023 & -0.03833276438 & $9.1858 \mathrm{e}-6$ & 0.04209103234 & 0.04210027036 & $9.2380 \mathrm{e}-6$ \\
\hline 0.7 & -0.0282878274 & -0.02827863504 & $9.1924 \mathrm{e}-6$ & 0.03203690952 & 0.03204614102 & $9.2315 \mathrm{e}-6$ \\
\hline 0.8 & -0.01823370458 & -0.01822450569 & $9.1989 \mathrm{e}-6$ & 0.0219827867 & 0.02199201168 & $9.2250 \mathrm{e}-6$ \\
\hline 0.9 & -0.008179581761 & -0.00817037635 & $9.2054 \mathrm{e}-6$ & 0.01192866388 & 0.01193788234 & $9.2185 e-6$ \\
\hline 1 & 0.00187454106 & 0.001883752993 & $9.2119 \mathrm{e}-6$ & 0.00187454106 & 0.001883752993 & $9.2119 e-6$ \\
\hline
\end{tabular}

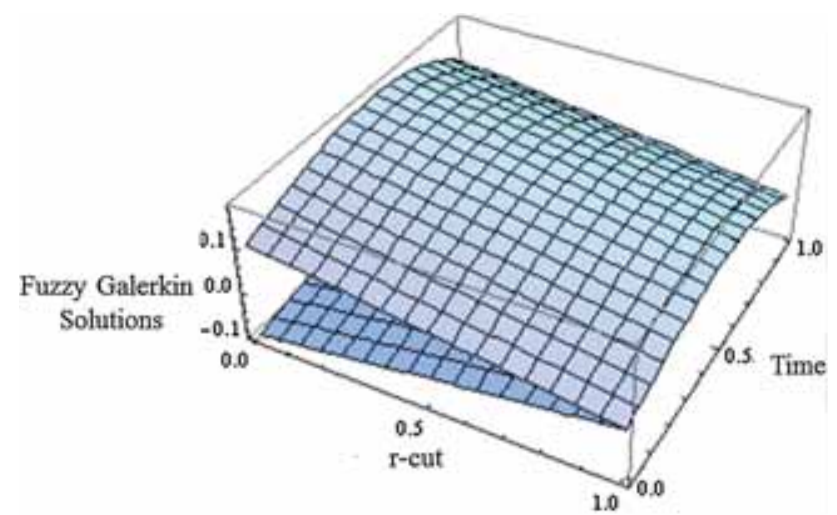

Figure 1. Fuzzy solution of Example 1 for Case 1 using the proposed method.

$$
\begin{aligned}
& \bar{Y}(t ; r)=t+(5-r) \cos (t)-(5-r) \cot (1) \sin (t) \\
& \quad+(1-r) \operatorname{cosec}(1) \sin (t) .
\end{aligned}
$$

Now, by using the proposed method we have $\widetilde{\phi}_{0}(t ; r)=[(1-t)(3+r)+t r,(1-t)(5-r)+(2-r) t]$.

Subsequently, by applying the procedure discussed previously, we get the following system of equations:

$\left[\begin{array}{cccccc}\frac{3}{10} & \frac{3}{20} & \frac{19}{210} & 0 & 0 & 0 \\ \frac{3}{20} & \frac{13}{105} & \frac{79}{840} & 0 & 0 & 0 \\ \frac{19}{210} & \frac{79}{840} & \frac{103}{1260} & 0 & 0 & 0 \\ 0 & 0 & 0 & \frac{3}{10} & \frac{3}{20} & \frac{19}{210} \\ 0 & 0 & 0 & \frac{3}{20} & \frac{13}{105} & \frac{79}{840} \\ 0 & 0 & 0 & \frac{19}{210} & \frac{79}{840} & \frac{103}{1260}\end{array}\right]\left\{\begin{array}{l}\underline{c}_{1} \\ \underline{c}_{2} \\ \underline{c}_{3} \\ \bar{c}_{1} \\ \bar{c}_{2} \\ \bar{c}_{3}\end{array}\right\}$

$=\left[\begin{array}{c}\frac{1}{6}+\frac{r}{6} \\ \frac{1}{20}+\frac{r}{12} \\ \frac{1}{60}+\frac{r}{20} \\ \frac{1}{2}-\frac{r}{6} \\ \frac{13}{60}-\frac{r}{12} \\ \frac{7}{60}-\frac{r}{20}\end{array}\right]$ 
Table 3. Fuzzy solution of Example 2 at $t=0.1$.

\begin{tabular}{|c|c|c|c|c|c|c|}
\hline$r$ & $\underline{y}(t ; r)$ & $\underline{Y}(t ; r)$ & Error & $\bar{y}(t ; r)$ & $\bar{Y}(t ; r)$ & Error \\
\hline 0 & 2.77412851 & 2.774064053 & $6.4457 e-5$ & 4.873214797 & 4.873150872 & $6.3925 \mathrm{e}-5$ \\
\hline 0.1 & 2.879082824 & 2.879018394 & $6.4430 \mathrm{e}-5$ & 4.768260483 & 4.768196531 & $6.3952 \mathrm{e}-5$ \\
\hline 0.2 & 2.984037138 & 2.983972735 & $6.4403 e-5$ & 4.663306169 & 4.66324219 & $6.3979 \mathrm{e}-5$ \\
\hline 0.3 & 3.088991453 & 3.088927076 & $6.4377 e-5$ & 4.558351854 & 4.558287849 & $6.4005 \mathrm{e}-5$ \\
\hline 0.4 & 3.193945767 & 3.193881417 & $6.4350 \mathrm{e}-5$ & 4.45339754 & 4.453333508 & $6.4032 \mathrm{e}-5$ \\
\hline 0.5 & 3.298900082 & 3.298835758 & $6.4324 \mathrm{e}-5$ & 4.348443225 & 4.348379167 & $6.4058 \mathrm{e}-5$ \\
\hline 0.6 & 3.403854396 & 3.403790099 & $6.4297 e-5$ & 4.243488911 & 4.243424826 & $6.4085 e-5$ \\
\hline 0.7 & 3.50880871 & 3.50874444 & $6.4270 \mathrm{e}-5$ & 4.138534597 & 4.138470485 & $6.4112 \mathrm{e}-5$ \\
\hline 0.8 & 3.613763025 & 3.613698781 & $6.4244 \mathrm{e}-5$ & 4.033580282 & 4.033516144 & $6.4138 \mathrm{e}-5$ \\
\hline 0.9 & 3.718717339 & 3.718653122 & $6.4217 \mathrm{e}-5$ & 3.928625968 & 3.928561803 & $6.4165 \mathrm{e}-5$ \\
\hline 1 & 3.823671653 & 3.823607463 & $6.4190 \mathrm{e}-5$ & 3.823671653 & 3.823607463 & $6.4190 \mathrm{e}-5$ \\
\hline
\end{tabular}

Table 4. Fuzzy solution of Example 2 at $t=0.01$.

\begin{tabular}{|c|c|c|c|c|c|c|}
\hline$r$ & $\underline{y}(t ; r)$ & $\underline{Y}(t ; r)$ & Error & $\bar{y}(t ; r)$ & $\bar{Y}(t ; r)$ & Error \\
\hline 0 & 2.978725494 & 2.978703791 & $2.1703 \mathrm{e}-5$ & 4.989550726 & 4.989529659 & $2.1067 \mathrm{e}-5$ \\
\hline 0.1 & 3.079266755 & 3.079245084 & $2.1671 \mathrm{e}-5$ & 4.889009464 & 4.888988366 & $2.1098 \mathrm{e}-5$ \\
\hline 0.2 & 3.179808017 & 3.179786378 & $2.1639 \mathrm{e}-5$ & 4.788468202 & 4.788447073 & $2.1129 \mathrm{e}-5$ \\
\hline 0.3 & 3.280349279 & 3.280327671 & $2.1608 \mathrm{e}-5$ & 4.687926941 & 4.687905779 & $2.1162 \mathrm{e}-5$ \\
\hline 0.4 & 3.38089054 & 3.380868965 & $2.1575 \mathrm{e}-5$ & 4.587385679 & 4.587364486 & $2.1193 \mathrm{e}-5$ \\
\hline 0.5 & 3.481431802 & 3.481410258 & $2.1544 \mathrm{e}-5$ & 4.486844418 & 4.486823192 & $2.1226 \mathrm{e}-5$ \\
\hline 0.6 & 3.581973063 & 3.581951551 & $2.1512 \mathrm{e}-5$ & 4.386303156 & 4.386281899 & $2.1257 \mathrm{e}-5$ \\
\hline 0.7 & 3.682514325 & 3.682492845 & $2.1480 \mathrm{e}-5$ & 4.285761895 & 4.285740605 & $2.1290 \mathrm{e}-5$ \\
\hline 0.8 & 3.783055587 & 3.783034138 & $2.1449 \mathrm{e}-5$ & 4.185220633 & 4.185199312 & $2.1321 \mathrm{e}-5$ \\
\hline 0.9 & 3.883596848 & 3.883575432 & $2.1416 \mathrm{e}-5$ & 4.084679371 & 4.084658019 & $2.1352 \mathrm{e}-5$ \\
\hline 1 & 3.98413811 & 3.984116725 & $2.1385 \mathrm{e}-5$ & 3.98413811 & 3.984116725 & $2.1385 \mathrm{e}-5$ \\
\hline
\end{tabular}

On solving the above system we have obtained the fuzzy constants $\widetilde{c}_{i}$ for $i=1, \ldots 3$ and putting these values in Eq. (39) we have

$$
\begin{aligned}
& \underline{y}(t ; r)=(1-t)(3+r)+t r+t(1-t) \\
& \quad \times\left(\frac{14(2332+1435 r)}{36777}-\frac{14(-557+41 r) t}{12259}-\frac{14}{299}(1+r) t^{2}\right), \\
& \bar{y}(t ; r)=(1-t)(5-r)+(2-r) t+t(1-t) \\
& \quad \times\left(-\frac{14(-5202+1435 r)}{36777}-\frac{14(475+41 r) t}{12259}+\frac{14}{299}(-3+r) t^{2}\right) .
\end{aligned}
$$

Again, it may be worth mentioning that the results obtained by proposed method are compared with exact solution for different values of $t$, which are tabulated in tables 3 and 4. Plots for Example 2 are depicted in figure 2.

Example 3 Consider the following FDE (Case 2):

$$
\widetilde{y}^{\prime \prime}-\widetilde{y}=1
$$

subject to the fuzzy boundary conditions as

$$
\begin{gathered}
\widetilde{y}(0)=[r-1,1-r], \widetilde{y}(0)=[r-1,1-r], \\
\widetilde{y}(1)=[r+(e-2), e-r] .
\end{gathered}
$$

We have obtained the exact fuzzy solution as follows:

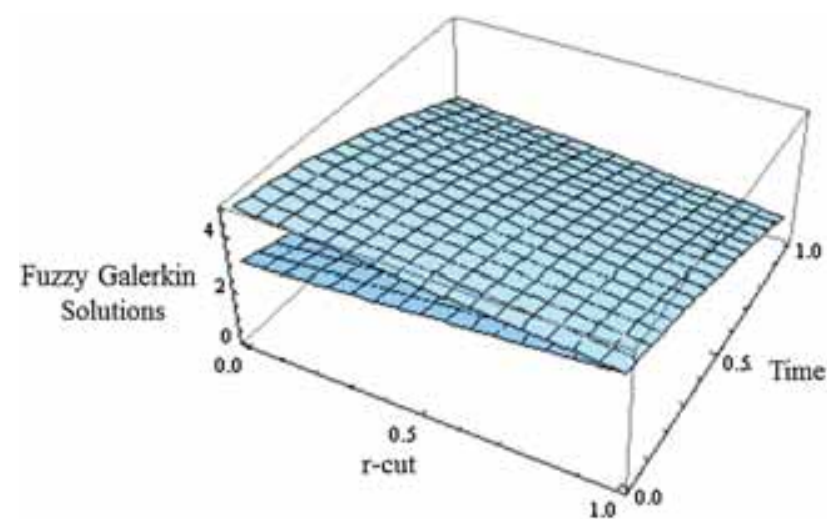

Figure 2. Fuzzy solution of Example 2 using the proposed method.

$\underline{Y}(t ; r)=-1+e^{t}+(r-1) \cos (t)+(r-1) \sin (t) \tan \left(\frac{1}{2}\right)$
$\bar{Y}(t ; r)=-1+e^{t}+(1-r) \cos (t)+(1-r) \sin (t) \tan \left(\frac{1}{2}\right)$.

Next, by following the proposed method we have 
$\begin{aligned} \widetilde{\phi}_{0}(t ; r)= & {[(1-t)(r-1)+t(r+(e-2)),(1-t)(1-r)} \\ & +t(e-r)] .\end{aligned}$

The following system is now obtained as

$$
\left[\begin{array}{cccccc}
\frac{1}{3} & \frac{1}{6} & \frac{1}{10} & \frac{1}{30} & \frac{1}{60} & \frac{1}{105} \\
\frac{1}{6} & \frac{2}{15} & \frac{1}{10} & \frac{1}{60} & \frac{1}{105} & \frac{1}{168} \\
\frac{1}{10} & \frac{1}{10} & \frac{3}{35} & \frac{1}{105} & \frac{1}{168} & \frac{1}{252} \\
\frac{1}{30} & \frac{1}{60} & \frac{1}{105} & \frac{1}{3} & \frac{1}{6} & \frac{1}{10} \\
\frac{1}{60} & \frac{1}{105} & \frac{1}{168} & \frac{3}{20} & \frac{13}{105} & \frac{79}{840} \\
\frac{1}{105} & \frac{1}{168} & \frac{1}{252} & \frac{1}{10} & \frac{1}{10} & \frac{3}{35}
\end{array}\right]\left\{\begin{array}{l}
\underline{c}_{1} \\
\underline{c}_{2} \\
\bar{c}_{1} \\
\bar{c}_{2} \\
\bar{c}_{3}
\end{array}\right\}
$$

And the solution may be found to be

$$
\begin{aligned}
& \underline{y}(t)=(1-t)(r-1)+t(r+e-2)+t(1-t) \\
& \left(\begin{array}{l}
-\frac{7}{86476182}(10608211+1848717 e-6748420 r) \\
-\frac{14}{14412697}(-138672+148304 e-48203 r) t \\
-\frac{7}{335179}(-1345+897 e+2242 r) t^{2}
\end{array}\right) \\
& \bar{y}(t)=(1-t)(1-r)+t(e-r)+t(t-1) \\
& \left(\begin{array}{l}
-\frac{7}{86476182}(-2888629+1848717 e+6748420 r) \\
-\frac{14}{14412697}(-235078+148304 e+48203 r) t \\
-\frac{7}{335179}(3139+897 e-2242 r) t^{2}
\end{array}\right)
\end{aligned}
$$

Again the solution obtained by proposed method is compared with the exact solution. Results are given in tables 5 and 6 , and plots for this example are shown in figure 3 .

Example 4 Next, we consider the following FDE (Case 3):

\begin{tabular}{|c|c|c|c|c|c|c|}
\hline$r$ & $\underline{y}(t ; r)$ & $\underline{Y}(t ; r)$ & Error & $\bar{y}(t ; r)$ & $\bar{Y}(t ; r)$ & Error \\
\hline 0 & -0.9443985268 & -0.9443724913 & $2.6036 \mathrm{e}-5$ & 1.154687761 & 1.154714327 & $2.6566 \mathrm{e}-5$ \\
\hline 0.1 & -0.8394442125 & -0.8394181503 & $2.6062 \mathrm{e}-5$ & 1.049733446 & 1.049759987 & $2.6541 \mathrm{e}-5$ \\
\hline 0.2 & -0.7344898981 & -0.7344638094 & $2.6089 \mathrm{e}-5$ & 0.944779132 & 0.9448056456 & $2.6514 \mathrm{e}-5$ \\
\hline 0.3 & -0.6295355837 & -0.6295094685 & $2.6115 \mathrm{e}-5$ & 0.8398248176 & 0.8398513046 & $2.6487 \mathrm{e}-5$ \\
\hline 0.4 & -0.5245812693 & -0.5245551275 & $2.6142 \mathrm{e}-5$ & 0.7348705033 & 0.7348969637 & $2.6460 \mathrm{e}-5$ \\
\hline 0.5 & -0.4196269549 & -0.4196007866 & $2.6168 \mathrm{e}-5$ & 0.6299161889 & 0.6299426228 & $2.6434 \mathrm{e}-5$ \\
\hline 0.6 & -0.3146726406 & -0.3146464457 & $2.6195 \mathrm{e}-5$ & 0.5249618745 & 0.5249882818 & $2.6407 \mathrm{e}-5$ \\
\hline 0.7 & -0.2097183262 & -0.2096921047 & $2.6222 \mathrm{e}-5$ & 0.4200075601 & 0.4200339409 & $2.6381 \mathrm{e}-5$ \\
\hline 0.8 & -0.1047640118 & -0.1047377638 & $2.6248 \mathrm{e}-5$ & 0.3150532457 & 0.3150795999 & $2.6354 \mathrm{e}-5$ \\
\hline 0.9 & 0.0001903025858 & 0.0002165771 & $2.6275 \mathrm{e}-5$ & 0.2100989313 & 0.210125259 & $2.6328 \mathrm{e}-5$ \\
\hline 1 & 0.105144617 & 0.1051709181 & $2.6301 \mathrm{e}-5$ & 0.105144617 & 0.1051709181 & $2.6301 \mathrm{e}-5$ \\
\hline
\end{tabular}

$$
\widetilde{y}^{\prime \prime}-3 \widetilde{y}^{\prime}+2 \widetilde{y}=0
$$

subject to the fuzzy boundary conditions

$$
\begin{aligned}
& \widetilde{y}(0)=[r+1,3-r], \\
& \widetilde{y}(1)=[r+2,4-r] .
\end{aligned}
$$

Table 5. Fuzzy solution of Example 2 at $t=0.1$. 
Table 6. Fuzzy solution of Example 2 at $t=0.01$.

\begin{tabular}{|c|c|c|c|c|c|c|}
\hline$r$ & $\underline{y}(t ; r)$ & $\underline{Y}(t ; r)$ & Error & $\bar{y}(t ; r)$ & $\bar{Y}(t ; r)$ & Error \\
\hline 0 & -0.9953709952 & -0.9953627672 & $8.2280 \mathrm{e}-6$ & 1.015454236 & 1.015463101 & $8.8650 \mathrm{e}-6$ \\
\hline 0.1 & -0.8948297336 & -0.8948214738 & $8.2598 \mathrm{e}-6$ & 0.9149129749 & 0.9149218079 & $8.8330 \mathrm{e}-6$ \\
\hline 0.2 & -0.794288472 & -0.7942801803 & $8.2917 \mathrm{e}-6$ & 0.8143717133 & 0.8143805145 & $8.8012 \mathrm{e}-6$ \\
\hline 0.3 & -0.6937472105 & -0.6937388869 & $8.3236 \mathrm{e}-6$ & 0.7138304517 & 0.7138392211 & $8.7694 \mathrm{e}-6$ \\
\hline 0.4 & -0.5932059489 & -0.5931975935 & $8.3554 \mathrm{e}-6$ & 0.6132891901 & 0.6132979276 & $8.7375 e-6$ \\
\hline 0.5 & -0.4926646873 & -0.4926563 & $8.3873 e-6$ & 0.5127479286 & 0.5127566342 & $8.7056 \mathrm{e}-6$ \\
\hline 0.6 & -0.3921234257 & -0.3921150066 & $8.4191 \mathrm{e}-6$ & 0.412206667 & 0.4122153408 & $8.6738 \mathrm{e}-6$ \\
\hline 0.7 & -0.2915821641 & -0.2915737132 & $8.4509 \mathrm{e}-6$ & 0.3116654054 & 0.3116740474 & $8.6420 \mathrm{e}-6$ \\
\hline 0.8 & -0.1910409025 & -0.1910324198 & $8.4827 \mathrm{e}-6$ & 0.2111241438 & 0.2111327539 & $8.6101 \mathrm{e}-6$ \\
\hline 0.9 & -0.09049964095 & -0.09049112634 & $8.5146 \mathrm{e}-6$ & 0.1105828822 & 0.1105914605 & $8.5783 \mathrm{e}-6$ \\
\hline 1 & 0.01004162064 & 0.01005016708 & $8.5464 \mathrm{e}-6$ & 0.01004162064 & 0.010050167 & $8.5464 \mathrm{e}-6$ \\
\hline
\end{tabular}

The exact fuzzy solution for this problem may be obtained as follows:

$$
\begin{gathered}
\underline{Y}(t ; r)=\frac{e^{-1-2 t}\left(-3 e^{3 t}+3 e^{4 t}+2 e^{2+3 t}-2 e^{1+4 t}\right)}{-1+e} \\
+\frac{(1-r) e^{-1-2 t}\left(-e^{2}+e^{3}+e^{1+t}-e^{3+t}\right)}{-1+e}, \\
\bar{Y}(t ; r)=\frac{e^{-1-2 t}\left(-3 e^{3 t}+3 e^{4 t}+2 e^{2+3 t}-2 e^{1+4 t}\right)}{-1+e} \\
+\frac{(r-1) e^{-1-2 t}\left(-e^{2}+e^{3}+e^{1+t}-e^{3+t}\right)}{-1+e} .
\end{gathered}
$$

Here we may have $\widetilde{\phi}_{0}(t ; r)=[(1-t)(r+1)+t(r+2)$, $(1-t)(3-r)+t(4-r)]$ and

$$
\left.\begin{array}{c}
{\left[\begin{array}{cccccc}
\frac{4}{15} & \frac{2}{15} & \frac{17}{210} & 0 & \frac{1}{20} & \frac{1}{20} \\
\frac{2}{15} & \frac{4}{35} & \frac{37}{420} & \frac{1}{20} & 0 & \frac{1}{70} \\
\frac{17}{210} & \frac{37}{420} & \frac{7}{90} & \frac{1}{20} & \frac{1}{70} & 0 \\
0 & \frac{1}{20} & \frac{1}{20} & \frac{4}{15} & \frac{2}{15} & \frac{17}{210} \\
\frac{1}{20} & 0 & \frac{1}{70} & \frac{2}{15} & \frac{4}{35} & \frac{37}{420} \\
\frac{1}{20} & \frac{1}{70} & 0 & \frac{17}{210} & \frac{37}{420} & \frac{7}{90}
\end{array}\right]\left\{\begin{array}{c}
\underline{c}_{1} \\
\underline{c}_{2} \\
\underline{c}_{3} \\
\bar{c}_{1} \\
\bar{c}_{2} \\
\bar{c}_{3}
\end{array}\right\}} \\
\frac{r}{3} \\
\frac{1}{60}+\frac{r}{6} \\
\frac{1}{60}+\frac{r}{10} \\
\frac{2}{3}-\frac{r}{3} \\
\frac{7}{20}-\frac{r}{6} \\
\frac{13}{60}-\frac{r}{10}
\end{array}\right] .
$$

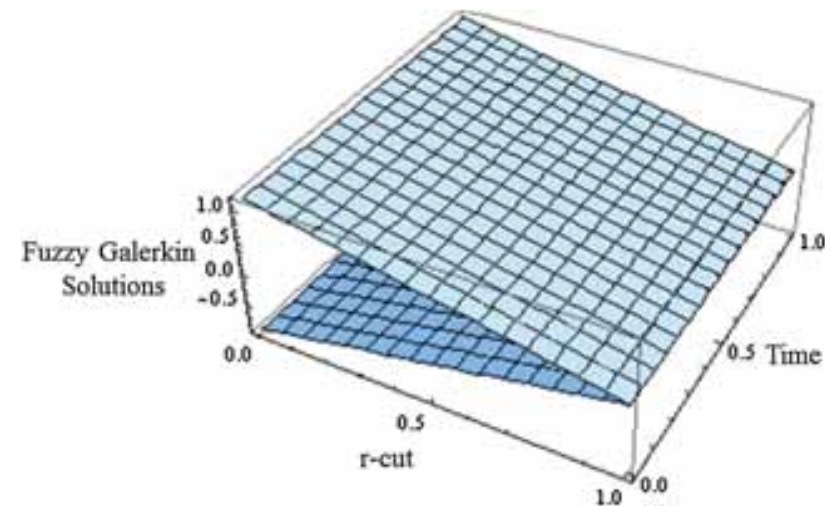

Figure 3. Fuzzy solution of Example 3 for Case 2 using the proposed method.

Hence the solutions are

$$
\begin{aligned}
& \underline{y}(t ; r)=(1+r)(1-t)+(2+r) t+(1-t) t \\
& \left(\frac{7(-1709+2434 r)}{10022}-\frac{7(-3079+2394 r) t}{10022}\right. \\
& \left.\quad+\frac{21(105+298 r) t^{2}}{10022}\right), \\
& \bar{y}(t ; r)=(3+r)(1-t)+(4-r) t+(1-t) t \\
& \left(-\frac{7(-3159+2434 r)}{10022}+\frac{7(-1709+2394 r) t}{10022}\right. \\
& \left.-\frac{21(-701+298 r) t^{2}}{10022}\right) .
\end{aligned}
$$

Here also above results are compared with the exact solution and are incorporated in tables 7 and 8 . Corresponding plots for this example are shown in figure 4.

Example 5 Let us now consider the following FDE (Case 2):

$$
\widetilde{y}^{\prime \prime \prime}-t \widetilde{y}=\left(t^{3}-2 t^{2}-5 t-3\right) e^{t}, 0 \leq t \leq 1
$$

subject to the fuzzy boundary conditions 
Table 7. Fuzzy solution of Example 4 at $t=0.1$.

\begin{tabular}{llccccc}
\hline$r$ & $\underline{y}(t ; r)$ & $\underline{Y}(t ; r)$ & Error & $\bar{y}(t ; r)$ & $\bar{Y}(t ; r)$ & \multicolumn{1}{c}{ Error } \\
\hline 0 & 1.012122481 & 1.010808546 & 0.0013 & 3.28915904 & 3.288607747 & $5.5129 \mathrm{e}-4$ \\
0.1 & 1.125974309 & 1.124698506 & 0.0013 & 3.175307212 & 3.174717787 & $5.8942 \mathrm{e}-4$ \\
0.2 & 1.239826136 & 1.238588466 & 0.0012 & 3.061455384 & 3.060827827 & $6.2756 \mathrm{e}-4$ \\
0.3 & 1.353677964 & 1.352478426 & 0.0012 & 2.947603556 & 2.946937867 & $6.6569 \mathrm{e}-4$ \\
0.4 & 1.467529792 & 1.466368386 & 0.0012 & 2.833751728 & 2.833047907 & $7.0382 \mathrm{e}-4$ \\
0.5 & 1.58138162 & 1.580258346 & 0.0011 & 2.7198999 & 2.719157947 & $7.4195 \mathrm{e}-4$ \\
0.6 & 1.695233448 & 1.694148306 & 0.0011 & 2.606048072 & 2.605267987 & $7.8009 \mathrm{e}-4$ \\
0.7 & 1.809085276 & 1.808038266 & 0.0010 & 2.492196244 & 2.491378027 & $8.1822 \mathrm{e}-4$ \\
0.8 & 1.922937104 & 1.921928226 & 0.0010 & 2.378344416 & 2.377488067 & $8.5635 \mathrm{e}-4$ \\
0.9 & 2.036788932 & 2.035818186 & $9.7075 \mathrm{e}-4$ & 2.264492588 & 2.263598107 & $8.9448 \mathrm{e}-4$ \\
1 & 2.15064076 & 2.149708147 & $9.3261 \mathrm{e}-004$ & 2.15064076 & 2.149708147 & $9.3261 \mathrm{e}-004$ \\
\hline
\end{tabular}

Table 8. Fuzzy solution of Example 4 at $t=0.01$.

\begin{tabular}{|c|c|c|c|c|c|c|}
\hline$r$ & $\underline{y}(t ; r)$ & $\underline{Y}(t ; r)$ & Error & $\bar{y}(t ; r)$ & $\bar{Y}(t ; r)$ & Error \\
\hline 0 & 0.9983957523 & 0.9979767927 & $4.1896 \mathrm{e}-4$ & 3.031727094 & 3.031632921 & $9.4173 e-5$ \\
\hline 0.1 & 1.100062319 & 1.099659599 & $4.0272 \mathrm{e}-4$ & 2.930060527 & 2.929950115 & $1.1041 \mathrm{e}-4$ \\
\hline 0.2 & 1.201728887 & 1.201342406 & $3.8648 \mathrm{e}-4$ & 2.82839396 & 2.828267308 & $1.2665 \mathrm{e}-4$ \\
\hline 0.3 & 1.303395454 & 1.303025212 & $3.7024 \mathrm{e}-4$ & 2.726727393 & 2.726584502 & $1.4289 \mathrm{e}-4$ \\
\hline 0.4 & 1.405062021 & 1.404708018 & $3.5400 \mathrm{e}-4$ & 2.625060826 & 2.624901695 & $1.5913 \mathrm{e}-4$ \\
\hline 0.5 & 1.506728588 & 1.506390825 & $3.3776 \mathrm{e}-4$ & 2.523394259 & 2.523218889 & $1.7537 \mathrm{e}-4$ \\
\hline 0.6 & 1.608395155 & 1.608073631 & $3.2152 \mathrm{e}-4$ & 2.421727692 & 2.421536083 & $1.9161 \mathrm{e}-4$ \\
\hline 0.7 & 1.710061722 & 1.709756438 & $3.0528 \mathrm{e}-4$ & 2.320061124 & 2.319853276 & $2.0785 \mathrm{e}-4$ \\
\hline 0.8 & 1.811728289 & 1.811439244 & $2.8904 \mathrm{e}-4$ & 2.218394557 & 2.21817047 & $2.2409 \mathrm{e}-4$ \\
\hline 0.9 & 1.913394856 & 1.91312205 & $2.7281 \mathrm{e}-004$ & 2.11672799 & 2.116487663 & $2.4033 \mathrm{e}-4$ \\
\hline 1 & 2.015061423 & 2.014804857 & $2.5657 \mathrm{e}-4$ & 2.015061423 & 2.014804857 & $2.5657 \mathrm{e}-4$ \\
\hline
\end{tabular}

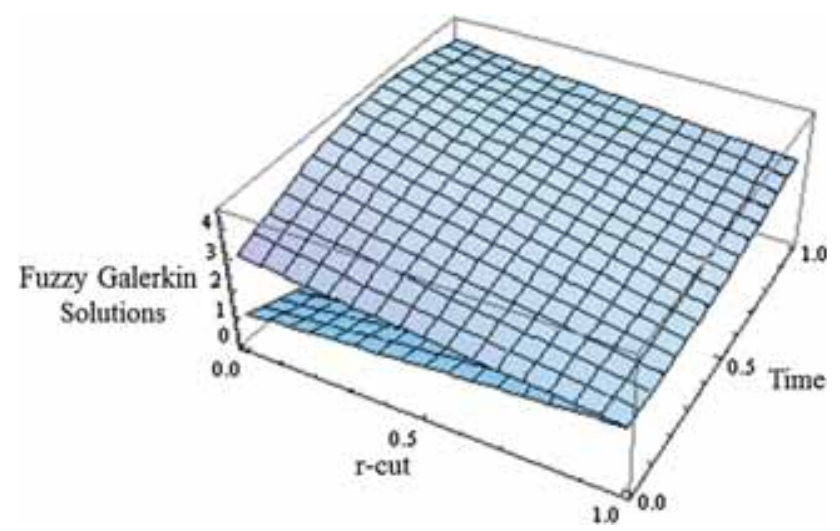

Figure 4. Fuzzy solution of Example 4 for Case 3 using the proposed method.

$$
\begin{gathered}
\widetilde{y}(0)=[0.2 r-0.2,0.2-0.2 r], \\
\widetilde{y}^{\prime}(0)=[0.2 r+0.8,1.2-0.2 r], \\
\widetilde{y}(1)=[0.2 r-0.2,0.2-0.2 r] .
\end{gathered}
$$

Here we may have $\widetilde{\phi}_{0}(t ; r)=[(0.2 r-0.2)+t(1-t)$ $(0.2 r+0.8),(0.2-0.2 r)+t(1-t)(1.2-0.2 r)] \quad$ and applying the procedure as discussed in section 3, one may have

$$
\begin{aligned}
& \underline{y}(t ; r)=(0.2 r-0.2)+(0.8+0.2 r)(1-t) t+t(1-t) \\
& \times\left\{\begin{array}{r}
(-8.27196-0.21426 r) \\
+t(1.06088+0.00826 r) \\
+t^{2}(0.57229+0.00819 r) \\
+t^{3}(0.20881+0.00009)
\end{array}\right\}, \\
& \bar{y}(t ; r)=(0.2-0.2 r)+(1.2-0.2 r)(1-t) t+t(1-t) \\
& \quad \times\left\{\begin{array}{r}
(-8.70050+0.21426 r) \\
+t(1.07710-0.00826 r) \\
+t^{2}(0.58867-0.00819 r) \\
+t^{3}(0.20900-0.00009)
\end{array}\right\} .
\end{aligned}
$$

Here also above results are compared with the special case $r=1$ [26] and are incorporated in table 9. Corresponding plots for this example are shown in figure 5 .

Example 6 In this example we have considered a 1-mm diameter, 50-mm long aluminium pin fin as shown in 
Table 9. Fuzzy solution of Example 4.

\begin{tabular}{|c|c|c|c|c|c|c|c|}
\hline \multirow[b]{2}{*}{$t$} & \multicolumn{2}{|c|}{$r=0.5$} & \multicolumn{2}{|c|}{$r=0.9$} & \multicolumn{2}{|c|}{$r=1$} & \multirow[b]{2}{*}{$Y(t ; r)[26]$} \\
\hline & $\underline{y}(t ; r)$ & $\bar{y}(t ; r)$ & $\underline{y}(t ; r)$ & $\bar{y}(t ; r)$ & & & \\
\hline 0 & -0.1 & 0.1 & -0.02 & 0.02 & 0 & 0 & 0 \\
\hline 0.1 & -0.763 & -0.5642 & -0.6835 & -0.6437 & -0.6636 & -0.6636 & 0.09947 \\
\hline 0.2 & -1.259 & -1.061 & -1.179 & -1.14 & -1.16 & -1.16 & 0.1954 \\
\hline 0.3 & -1.591 & -1.394 & -1.512 & -1.473 & -1.493 & -1.493 & 0.2835 \\
\hline 0.4 & -1.767 & -1.57 & -1.688 & -1.649 & -1.669 & -1.669 & 0.358 \\
\hline 0.5 & -1.794 & -1.596 & -1.715 & -1.675 & -1.695 & -1.695 & 0.4122 \\
\hline 0.6 & -1.681 & -1.483 & -1.602 & -1.562 & -1.582 & -1.582 & 0.4373 \\
\hline 0.7 & -1.44 & -1.241 & -1.36 & -1.32 & -1.34 & -1.34 & 0.4229 \\
\hline 0.8 & -1.084 & -0.8846 & -1.004 & -0.9644 & -0.9844 & -0.9844 & 0.3561 \\
\hline 0.9 & -0.6311 & -0.4311 & -0.5511 & -0.5111 & -0.5311 & -0.5311 & 0.2214 \\
\hline 1 & -0.1 & 0.1 & -0.02 & 0.02 & 0 & 0 & 0 \\
\hline
\end{tabular}

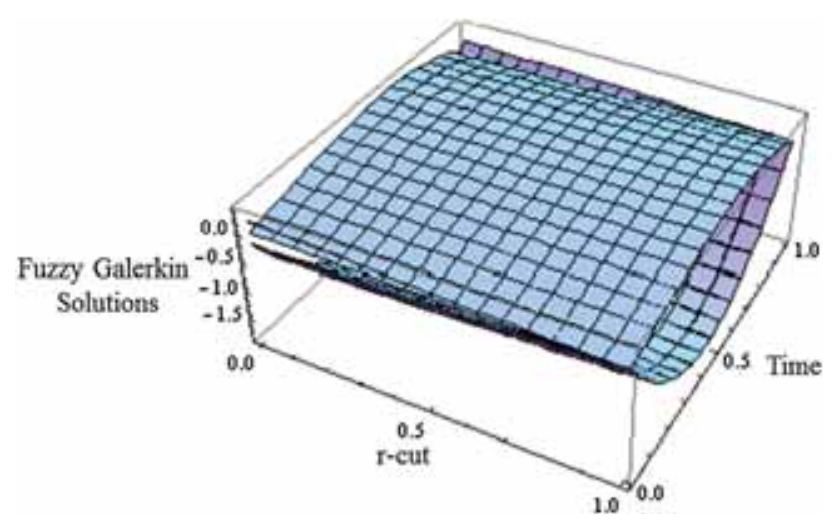

Figure 5. Fuzzy solution of Example 3 for Case 2 using the proposed method.

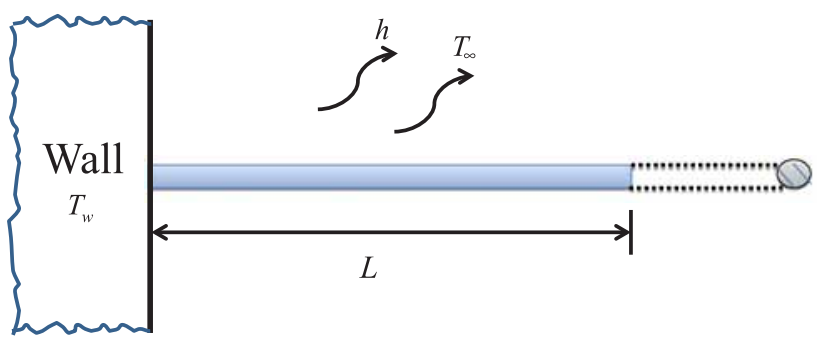

Figure 6. A pin-fin Seshu [27].

figure 6 used to enhance the heat transfer from a surface wall maintained at $300^{\circ} \mathrm{C}$. The governing differential equation is

$$
k \frac{d^{2} \widetilde{T}}{d x^{2}}=\frac{P h}{A_{c}}\left(\widetilde{T}-\widetilde{T}_{\infty}\right)
$$

subject to the fuzzy boundary conditions

$$
\begin{aligned}
& \widetilde{T}(0 ; r)=[20 r+280,320-20 r] \\
& \frac{d \widetilde{T}}{d x}(L)=[0.8 r-0.8,0.2-0.2 r] .
\end{aligned}
$$

Here the considered parameters are defined as below.

$k=$ coefficient of thermal conductivity,

$P=$ perimeter,

$A_{c}=$ cross-sectional area,

$h=$ convective heat transfer coefficient,

$\widetilde{T}_{w}=$ fuzzy wall temperature,

$\widetilde{T}_{\infty}=$ ambient temperature.

Let $k=200 \mathrm{~W} / \mathrm{m} /{ }^{\circ} \mathrm{C}$ for aluminium, $h=20 \mathrm{~W} / \mathrm{m}^{2}$ ${ }^{\circ} \mathrm{C}, \widetilde{T}_{\infty}=30^{\circ} \mathrm{C}$. Thus Eq. (43) is reduced to

$$
\frac{d^{2} \widetilde{T}}{d x^{2}}=400(\widetilde{T}-30) .
$$

The exact bounds of the fuzzy solutions are found as follows:

$$
\begin{aligned}
\underline{T}(x ; r)= & e^{-20 x}\left(220.212+30 e^{20 x}+29.7878 e^{40 x}\right. \\
& \left.+17.603 r+2.39702 e^{40 x} r\right), \\
\bar{T}(x ; r)= & e^{-20 x}\left(255.428+30 e^{20 x}+34.5721 e^{40 x}\right. \\
& \left.-17.6127 r-2.3873 e^{40 x} r\right) .
\end{aligned}
$$

Here we may have $\widetilde{\phi}_{0}(t ; r)=[(20 r+280)+$ $x(0.8 r 0.8),(320-20 r)+x(0.2-0.2 r)]$. Fuzzy solutions are obtained using the present procedure and are presented in tables 9-13. Tables 9 and 11 gives the lower and upper bounds of the fuzzy solutions, respectively, along with the comparison of Bede [9] for $r=0.6$ with different values of $x$. Also comparisons have been made in table 12 with the crisp solution obtained by Bede [9] and Seshu [27] for different values of $x$ and $r=1$. Similarly table 13 
Table 10. Comparison of various lower bound solutions for uncertain temperature in a fin for $r=0.6$.

\begin{tabular}{|c|c|c|c|c|c|c|}
\hline \multirow[b]{2}{*}{$x$} & \multicolumn{2}{|c|}{ Quadratic solution } & \multicolumn{2}{|c|}{ Cubic solution } & \multicolumn{2}{|c|}{ Quartic solution } \\
\hline & Present & Bede [9] & Present & Bede [9] & Present & Bede [9] \\
\hline 0 & 292 & 292 & 292 & 292 & 292 & 292 \\
\hline 0.005 & 274.28 & 273.32 & 273.300 & 273.300 & 273.32 & 273.300 \\
\hline 0.01 & 258.77 & 257.08 & 257.03 & 257.03 & 257.08 & 257.03 \\
\hline 0.015 & 245.42 & 243.11 & 243.02 & 243.02 & 243.11 & 243.02 \\
\hline 0.02 & 234.16 & 231.28 & 231.15 & 231.15 & 231.28 & 231.15 \\
\hline 0.025 & 224.95 & 221.45 & 221.29 & 221.29 & 221.45 & 221.29 \\
\hline 0.03 & 217.71 & 213.55 & 213.25 & 213.25 & 213.55 & 213.25 \\
\hline 0.035 & 212.40 & 207.48 & 207.24 & 207.24 & 207.48 & 207.24 \\
\hline 0.04 & 208.95 & 203.19 & 202.91 & 202.91 & 203.19 & 202.91 \\
\hline 0.045 & 207.31 & 200.63 & 200.31 & 200.31 & 200.63 & 200.31 \\
\hline 0.05 & 207.42 & 199.78 & 199.41 & 199.41 & 199.78 & 199.41 \\
\hline
\end{tabular}

Table 11. Comparison of various upper bound solutions for uncertain temperature in a fin for $r=0.6$.

\begin{tabular}{|c|c|c|c|c|c|c|}
\hline \multirow[b]{2}{*}{$x$} & \multicolumn{2}{|c|}{ Quadratic solution } & \multicolumn{2}{|c|}{ Cubic solution } & \multicolumn{2}{|c|}{ Quartic solution } \\
\hline & Present & Bede [9] & Present & Bede Bede [9] & Present & Bede [9] \\
\hline 0 & 308 & 308 & 308 & 308 & 308 & 308 \\
\hline 0.005 & 289.20 & 288.18 & 288.16 & 288.18 & 288.23 & 288.18 \\
\hline 0.01 & 272.74 & 270.95 & 270.89 & 270.95 & 271.05 & 270.95 \\
\hline 0.015 & 258.58 & 256.13 & 256.03 & 256.13 & 256.28 & 256.13 \\
\hline 0.02 & 246.64 & 243.57 & 243.44 & 243.57 & 243.77 & 243.57 \\
\hline 0.025 & 236.86 & 233.15 & 232.98 & 233.15 & 233.41 & 233.15 \\
\hline 0.03 & 229.18 & 224.77 & 224.55 & 224.77 & 225.08 & 224.77 \\
\hline 0.035 & 223.55 & 218.33 & 218.08 & 218.33 & 218.70 & 218.33 \\
\hline 0.04 & 219.89 & 213.78 & 213.48 & 213.78 & 214.21 & 213.78 \\
\hline 0.045 & 218.15 & 211.06 & 210.72 & 211.06 & 211.56 & 211.06 \\
\hline 0.05 & 218.27 & 210.16 & 209.77 & 210.16 & 210.73 & 210.16 \\
\hline
\end{tabular}

Table 12. Comparison of the solutions for uncertain temperature in a fin for $r=1$.

\begin{tabular}{|c|c|c|c|c|c|c|c|c|c|}
\hline \multirow[b]{2}{*}{$x$} & \multicolumn{3}{|c|}{ Quadratic solution } & \multicolumn{3}{|c|}{ Cubic solution } & \multicolumn{3}{|c|}{ Quartic solution } \\
\hline & Present & Bede [9] & Seshu [27] & Present & Bede [9] & Seshu [27] & Present & Bede [9] & Seshu [27] \\
\hline 0 & 300 & 300 & 300 & 300 & 300 & 300 & 300 & 300 & 300 \\
\hline 0.005 & 281.74 & 280.75 & 281.59 & 280.73 & 280.75 & 280.87 & 280.80 & 280.75 & 280.80 \\
\hline 0.01 & 265.76 & 264.02 & 265.12 & 263.96 & 264.02 & 264.11 & 264.11 & 264.02 & 264.11 \\
\hline 0.015 & 251.99 & 249.62 & 250.59 & 249.53 & 249.62 & 249.62 & 249.77 & 249.62 & 249.77 \\
\hline 0.02 & 240.40 & 237.43 & 238.00 & 237.29 & 237.43 & 237.33 & 237.62 & 237.43 & 237.62 \\
\hline 0.025 & 230.90 & 227.31 & 227.34 & 227.14 & 227.31 & 227.16 & 227.55 & 227.31 & 227.55 \\
\hline 0.03 & 223.45 & 219.16 & 218.62 & 218.95 & 219.16 & 219.02 & 219.46 & 219.16 & 219.46 \\
\hline 0.035 & 217.97 & 212.91 & 211.84 & 212.66 & 212.91 & 212.83 & 213.27 & 212.91 & 213.27 \\
\hline 0.04 & 214.42 & 208.49 & 207.00 & 208.20 & 208.49 & 208.51 & 208.90 & 208.49 & 208.90 \\
\hline 0.045 & 212.73 & 205.85 & 204.09 & 205.52 & 205.85 & 205.98 & 206.33 & 205.85 & 206.33 \\
\hline 0.05 & 212.85 & 204.97 & 203.12 & 204.59 & 204.97 & 204.16 & 204.65 & 204.97 & 204.91 \\
\hline
\end{tabular}

incorporates the solution bounds for $x=0.2$ with different values of $r$.

It is worth mentioning that the main value of the paper may not be the example problems as discussed above. Here the main contribution is the new Galerkin type method to handle $n$th order FDEs. As such some known FDEs are solved as test problems to have the confidence on the proposed method. The solutions by the proposed method in 
Table 13. Comparison of various bound solutions for uncertain temperature in a fin for $x=0.2$.

\begin{tabular}{|c|c|c|c|c|c|c|c|c|}
\hline \multirow[b]{2}{*}{$r$} & \multicolumn{2}{|c|}{$\begin{array}{c}\text { Quadratic solution } \\
\text { Present }\end{array}$} & \multicolumn{2}{|c|}{$\begin{array}{l}\text { Cubic solution } \\
\text { Present }\end{array}$} & \multicolumn{2}{|c|}{$\begin{array}{c}\text { Quartic solution } \\
\text { Present }\end{array}$} & \multicolumn{2}{|c|}{ Bede [9] } \\
\hline & $\underline{T}(x)$ & $\bar{T}(x)$ & $\underline{T}(x)$ & $\bar{T}(x)$ & $\underline{T}(x)$ & $\bar{T}(x)$ & $\underline{T}(x)$ & $\bar{T}(x)$ \\
\hline 0 & 224.81 & 255.99 & 221.93 & 252.65 & 222.23 & 253.00 & 222.05 & 252.79 \\
\hline 0.1 & 226.37 & 254.43 & 223.47 & 251.11 & 223.77 & 251.46 & 223.59 & 251.26 \\
\hline 0.2 & 227.93 & 252.87 & 225.00 & 249.58 & 225.31 & 249.92 & 225.13 & 249.72 \\
\hline 0.3 & 229.48 & 251.31 & 226.54 & 248.04 & 226.85 & 248.39 & 226.66 & 248.18 \\
\hline 0.4 & 231.04 & 249.75 & 228.08 & 246.51 & 228.39 & 246.85 & 228.20 & 246.65 \\
\hline 0.5 & 232.60 & 248.19 & 229.61 & 244.97 & 229.93 & 245.31 & 229.74 & 245.11 \\
\hline 0.6 & 234.16 & 246.63 & 231.15 & 243.44 & 231.46 & 243.77 & 231.28 & 243.57 \\
\hline 0.7 & 235.72 & 245.08 & 232.69 & 241.90 & 233.00 & 242.23 & 232.81 & 242.04 \\
\hline 0.8 & 237.28 & 243.52 & 234.22 & 240.37 & 234.54 & 240.70 & 234.35 & 240.50 \\
\hline 0.9 & 238.84 & 241.96 & 235.76 & 238.83 & 236.08 & 239.16 & 235.89 & 238.96 \\
\hline 1 & 240.40 & 240.40 & 237.29 & 237.29 & 237.62 & 237.62 & 237.43 & 237.43 \\
\hline
\end{tabular}

all the test problems are found to be very close to the exact solutions. Finally the proposed method has been applied to an application problem too. The proposed method may be found to be a straightforward and alternate way to handle $n$th order FBVPs.

\section{Conclusions}

In this paper, the Galerkin method has been successfully applied to find fuzzy solution of $n$th order fuzzy boundary value problems. The proposed methodology is applied for both positive and negative coefficient of the fuzzy differential equations. Also the obtained results are compared with the exact as well as other existing method(s) and are found to be in good agreement.

\section{Acknowledgements}

The first author would like to acknowledge the Grant No. 106112016CDJCR101208 sponsored by the Chongqing University, China under the scheme of special fundamental research projects for the central universities to carry out the present investigation. J J Nieto and J Losada also acknowledge partial financial support by the Ministerio de Economía y Competitividad of Spain under grant MTM2010-15314 and MTM2013-43014-P, XUNTA under grant R2014/002, and co-financed by the European Community fund FEDER.

\section{References}

[1] Zadeh L A 1965 Fuzzy Sets Inf. Control 8: 338-353

[2] Chang S L and Zadeh L A 1972 On fuzzy mapping and control. IEEE Trans. Syst. Man Cyber. 2: 30-34

[3] Dubois D and Prade H 1982 Towards fuzzy differential calculus, part 3: differentiation. Fuzzy Sets Syst. 8: 225-233

[4] Puri M L and Ralescu D A 1983 Differentials for fuzzy functions. J. Math. Anal. Appl. 91: 552-558
[5] Goetschel R and Voxman W 1986 Elementary calculus. Fuzzy Sets Syst. 18: 31-43

[6] Kaleva O 1987 Fuzzy differential equations. Fuzzy Sets Syst. 24: $301-317$

[7] Kaleva O 1990 The Cauchy problem for fuzzy differential equations. Fuzzy Sets Syst. 35: 389-396

[8] Seikkala S 1987 On the fuzzy initial value problem. Fuzzy Sets Syst. 24: 319-330

[9] Bede B 2008 Note on numerical solutions of fuzzy differential equations by predictor-corrector method. Inf. Sci. 178: 1917-1922

[10] Buckley J J and Feuring T 2001 Fuzzy initial value problem for $n$ th-order linear differential equations. Fuzzy Sets Syst. 121: 247-255

[11] Ahmada M Z, Hasan M K and Baets B D 2013 Analytical and numerical solutions of fuzzy differential equations. Inf. Sci. 236: 156-167

[12] Oregan D, Lakshmikantham V and Nieto J J 2003 Initial and boundary value problems for fuzzy differential equations. Nonlin. Anal. 54: 405-415

[13] Tapaswini S and Chakraverty S 2014 New analytical method for solving $n$-th order fuzzy differential equations. Ann. Fuzzy Math. Inf. 8: 231-244

[14] Chen M, Wu C, Xue X and Liu G 2008a On fuzzy boundary value problems. Inf. Sci. 178: 1877-1892

[15] Khastan A and Nieto J J 2010 A boundary value problem for second order fuzzy differential equations. Nonlin. Anal.: Theory Methods Appl. 72: 3583-3593

[16] Mohammed O H and Fadhel F S 2010 The collocation method for solving nonhomogeneous fuzzy boundary value problems. J. Al-Nahrain Univ. 13: 229-234

[17] Jamshidi L and Avazpour L 2012 Solution of the fuzzy boundary value differential equations under generalized differentiability by shooting method. J. Fuzzy Set Valued Anal. 2012: 1-19

[18] Guo X, Shang D and Lu X 2013 Fuzzy approximate solutions of second-order fuzzy linear boundary value problems. Bound. Value Problems 212: 1-17

[19] Dahalan A A, Muthuvalu M S and Sulaiman J 2013 Numerical solutions of two-point fuzzy boundary value problem using half-sweep alternating group explicit method. In: Proceedings of the International Conference on 
Mathematical Sciences and Statistics (ICMSS2013) 2013, AIP Conf. Proc. 1557: 103-107

[20] Jameel A F and Ismail A I 2013 Approximate solution of $N$ th order two point fuzzy boundary value problems by optimal homotopy asymptotic method. Int. J. Mod. Math. Sci. 6: 107-120

[21] Chen M, Fu Y, Xue X and Wu C 2008b Two-point boundary value problems of undamped uncertain dynamical systems. Fuzzy Sets Syst. 159: 2077-2089

[22] Hanss M 2005 Applied fuzzy arithmetic: an introduction with engineering applications. Berlin: Springer-Verlag
[23] Jaulin L, Kieffer M, Didri O T and Walter E 2001 Applied interval analysis. Springer

[24] Zimmermann H J 2001 Fuzzy set theory and its application. Boston/Dordrecht/London: Kluwer Academic Publishers

[25] Chalco-Cano Y and Roman-Flores H 2008 On new solutions of fuzzy differential equations. Chaos Solitons Fractals 38: 112-119

[26] Li Z, Wang Y and Tan F 2012 The solution of a class of third-order boundary value problems by the reproducing kernel method. Abstract Appl. Anal. 2012: 1-11

[27] Seshu P 2012 Text book of finite element analysis. PHI Publishers 